ARTICLE

https://doi.org/10.1038/s41467-020-20552-y

\title{
Overexpression of neuregulin 1 in GABAergic interneurons results in reversible cortical disinhibition
}

Yao-Yi Wang ${ }^{1,2}$, Bing Zhao ${ }^{1,2}$, Meng-Meng Wu ${ }^{1,2}$, Xiao-Li Zheng ${ }^{1}$, Longnian $\operatorname{Lin}^{1} \&$ Dong-Min Yin (1) ${ }^{1 凶}$

Cortical disinhibition is a common feature of several neuropsychiatric diseases such as schizophrenia, autism and intellectual disabilities. However, the underlying mechanisms are not fully understood. To mimic increased expression of $\mathrm{Nrg}$, a schizophrenia susceptibility gene in GABAergic interneurons from patients with schizophrenia, we generated gtoNrg1 mice with overexpression of $\mathrm{Nrg} 1$ in GABAergic interneurons. gtoNrg1 mice showed cortical disinhibition at the cellular, synaptic, neural network and behavioral levels. We revealed that the intracellular domain of NRG1 interacts with the cytoplasmic loop 1 of $\mathrm{Na}_{\mathrm{v}} 1.1$, a sodium channel critical for the excitability of GABAergic interneurons, and inhibits $\mathrm{Na}_{\mathrm{v}}$ currents. Intriguingly, activation of GABAergic interneurons or restoring NRG1 expression in adulthood could rescue the hyperactivity and impaired social novelty in gtoNrg1 mice. These results identify mechanisms underlying cortical disinhibition related to schizophrenia and raise the possibility that restoration of NRG1 signaling and GABAergic function is beneficial in certain neuropsychiatric disorders.

\footnotetext{
${ }^{1}$ Key Laboratory of Brain Functional Genomics, Ministry of Education and Shanghai, School of Life Science, East China Normal University, Shanghai, China.

${ }^{2}$ These authors contributed equally: Yao-Yi Wang, Bing Zhao, Meng-Meng Wu. ${ }^{\bowtie}$ email: dmyin@brain.ecnu.edu.cn
} 
C ortical disinhibition is a common feature of several neuropsychiatric diseases such as schizophrenia, autism, and intellectual disabilities ${ }^{1}$. However, the underlying molecular and cellular mechanisms are not fully understood. Neuregulin $1(\mathrm{Nrg} 1)$ is genetically associated with schizophrenia in diverse populations ${ }^{2-4}$. The Nrg1 gene encodes NRG1 protein, a trophic factor implicated in both neurodevelopment and neurotransmission ${ }^{5}$. NRG1 has over 30 splicing isoforms per the different amino acid sequences in the N-terminus ${ }^{6}$. The $\mathrm{C}$-terminal fragment of NRG1 (NRG1-CTF), which is generated by extracellular cleavage, can be further cleaved by $\gamma$-secretase to generate the NRG1-intracellular domain (NRG1-ICD) ${ }^{6}$. The amino acid sequences of NRG1-ICD are more conserved among different species than the $\mathrm{N}$-terminus ${ }^{7}$. Most of the single-nucleotide polymorphisms (SNPs) in the Nrgl gene that are associated with schizophrenia are localized in intronic, noncoding regions ${ }^{6}$, raising the possibility that they may regulate the expression of the Nrg1 gene. While some studies showed isoform 1 alpha of Nrg1 was lower in brains of schizophrenia patients 8,9 , other studies reported increased Nrg1 expression or elevated NRG1 signaling in schizophrenia brain ${ }^{10-14}$. Of note, the increase of $\mathrm{Nrg} 1$ expression in schizophrenia brain did not correlate with antipsychotic treatment ${ }^{11,13}$, suggesting an association with the disorder instead of medication.

$N r g 1$ is highly expressed in neurons in the mammalian brain ${ }^{15}$. Recent studies based on single-cell RNA sequencing revealed that Nrg1 was expressed in both inhibitory and excitatory neurons from mouse and human prefrontal cotex $(\mathrm{PFC})^{16,17}$, a key brain region implicated in schizophrenia ${ }^{18,19}$. Most postmortem studies analyzed the gene expression in the total homogenate of schizophrenia brain, likely masking cell-type-specific alterations due to cellular heterogeneity ${ }^{20}$. Two recent studies reported the transcriptome alterations in GABAegic interneurons (mainly were parvalbumin $^{+}$) and pyramidal neurons (PN) from the PFC of schizophrenia patients ${ }^{21,22}$. Here, we analyzed the cell-typespecific Nrg1 expression in the GEO database: GSE93577 and GSE9398721,22. We found that Nrg1 expression was increased in GABAergic interneurons but not in PN from the PFC of schizophrenia patients, compared with age- and sex-matched healthy controls.

To mimic increased Nrg1 expression in GABAergic interneurons from schizophrenia patients, we generated gtoNrg1 mice in which $\mathrm{Nrg} 1$ overexpression specifically occurred in GABAergic interneurons and could be turned off by doxycycline (Dox). Intriguingly, gtoNrg1 mice showed cortical disinhibition at the cellular, synaptic, and neural network levels. The mechanistic study suggested that NRG1-ICD could interact with $\mathrm{Na}_{\mathrm{v}} 1.1$, a sodium channel critical for the excitability of GABAergic interneurons and inhibit the $\mathrm{Na}_{\mathrm{v}}$ currents. We further demonstrated that cortical disinhibition led to behavioral deficits in gtoNrg1 mice. Lastly, both cortical disinhibition and behavioral deficits in gtoNrg1 mice disappeared when NRG1 expression returned to normal in adulthood. Together, our results demonstrate mechanisms underlying cortical disinhibition related to schizophrenia and shed light on the pathophysiology of neuropsychiatric disorders.

\section{Results}

Generation of gtoNrg1 mice to mimic increased Nrg1 expression in GABAergic interneurons from schizophrenia patients. To address the cell-type-specific alteration of Nrg1 gene expression in the postmortem PFC of schizophrenia patients, we analyzed the GEO database GSE9357721 and GSE9398722. The database GSE93577 was composed of gene expression data in GABAergic interneurons from 36 schizophrenia patients and 36 matched healthy controls (Supplementary Table 1). The database GSE93987 included the gene expression data in layer $3 \mathrm{PN}$ from the same PFC samples as database GSE93577 (Supplementary Table 1). Type I Nrg1 mRNA levels were significantly increased in GABAergic interneurons from schizophrenia PFC, compared with age- and sex-matched controls (Fig. 1a). By contrast, Nrg1 mRNA levels were not significantly altered in PN from schizophrenia PFC, compared to age- and sex-matched controls (Fig. 1b). The increased type I Nrg1 expression in GABAergic interneurons from schizophrenia PFC seems not to be due to the antipsychotic treatment because type I $\mathrm{Nrg} 1 \mathrm{mRNA}$ levels were similar between drug-naive and drug-treated schizophrenia patients (Supplementary Fig. 1a). There is no sex difference for type I Nrg1 expression in the schizophrenia PFC (Supplementary Fig. 1b). Type IV Nrg1 expression was not significantly increased in GABAergic interneurons from schizophrenia PFC (Supplementary Fig. 1c), suggesting a specificity for an increase of type I Nrg1 expression in GABAergic interneurons. Neuregulin 3 (Nrg3) is another schizophrenia risk factor among the neuregulin gene family ${ }^{23-25}$. By contrast, the mRNA levels of $\mathrm{Nrg3}$ were reduced in GABAergic interneurons from schizophrenia PFC, compared with age- and sex-matched controls (Supplementary Fig. 1d). Together, these results suggest that type I Nrg1 expression is increased in GABAergic interneurons from the postmortem PFC of schizophrenia patients.

To mimic the increased type I Nrgl expression in GABAergic interneurons from schizophrenia patients, we aim to generate Nrg1 transgenic mice that overexpress type I Nrg1 specifically in GABAergic interneurons. Toward this goal, TRE-Nrg1 mice (Fig. 1c) ${ }^{26}$ were crossed with Gad67-tTA mice which express tTA specifically in GABAergic interneurons ${ }^{27}$ (Supplementary Fig. 2). Resulting Gad67-tTA; TRE-Nrg1 mice (gtoNrg1 for Gad67 promoter-driven tet-off $\mathrm{Nrg1}$ ) produced HA-NRG1 in GABAergic interneurons in the absence of Dox (Fig. 1d). We used Gad67tTA mice as controls in the following experiments (see also the methods). As shown in Fig. 1e, the mRNA of Nrg1 was increased in several brain regions including olfactory bulb (OB), striatum (STR), and prefrontal cortex (PFC) in gtoNrg1 mice, compared with controls. The increase was due to the expression of the transgene, which was detectable by the expression of the HA tag (Fig. 1f). In agreement, the protein levels of NRG1 (including the full length and ICD) were increased by $50-100 \%$ in the PFC of gtoNrg1 mice (Fig. 1g, h). To demonstrate the Nrg1 transgene is specifically expressed in GABAergic interneurons in the PFC, we performed single-cell RT-PCR. As shown in Supplementary Fig. 1e, the Nrg1 transgene indicated by the expression of HA tag was expressed in GABAergic interneurons but not in PN. Lastly, overexpression of $\mathrm{Nrgl}$ could be switched off by feeding the mice with Dox-containing water (Fig. 1e-h). Together, the results indicate that gtoNrg1 mice express higher levels of $\mathrm{Nrgl}$ specifically in GABAergic interneurons, and the overexpression could be turned off efficiently by Dox.

Behavioral deficits in gtoNrg1 mice. gtoNrg1 mice showed similar body weight with control littermates (Supplementary Fig. 3a), indicating no growth retardation. However, they were hyperactive in the open-field test (Fig. 2a), a phenotype related to abnormalities of brain dopamine levels in schizophrenia patients. By contrast, the staying time in the margin and center of the open filed was similar between control and gtoNrg1 mice (Supplementary Fig. 3b), indicating no anxiety-like phenotype. Prepulse inhibition (PPI) is a common test of sensorimotor gating that is often impaired in schizophrenia patients ${ }^{28}$. gtoNrg1 mice had a normal response to $75-85 \mathrm{~dB}$ background noise (Supplementary Fig. $3 \mathrm{c}-\mathrm{e}$ ). The prepulse itself has significant effects on PPI in both control and 
a

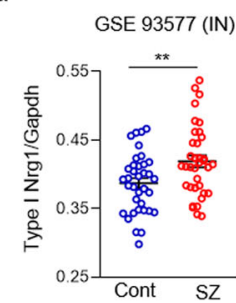

b

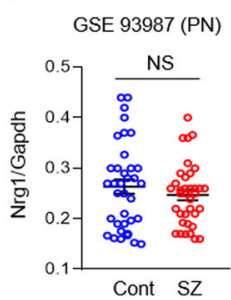

C

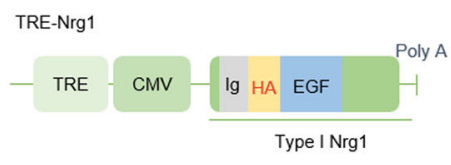

d



g

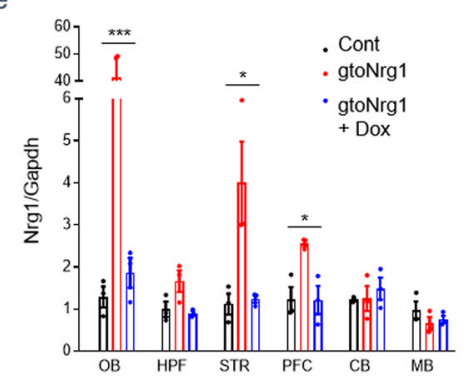

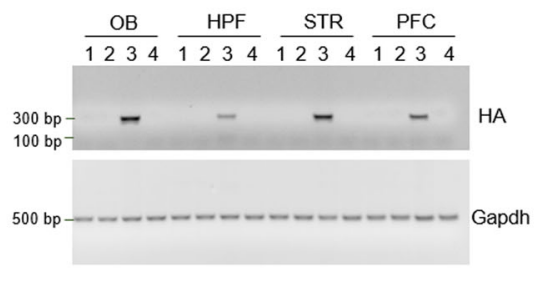

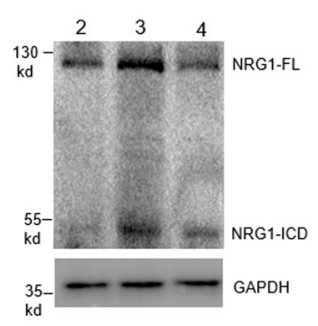

h
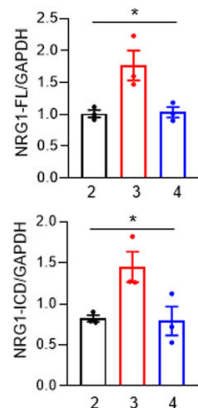

Fig. 1 Generation of gtoNrg1 mice to mimic increased $\mathbf{N r g 1}$ expression in GABAergic interneurons from schizophrenia patients. a Increased type I Nrg1 mRNA levels in GABAergic interneurons from schizophrenia PFC, compared with age- and sex-matched controls. ${ }^{\star \star} P=0.0064$, two-sided $t$ test, $n=36$ for each group. Data are presented as mean values + /- SEM. b Similar Nrg1 expression in pyramidal neurons from layer 3 PFC between control and schizophrenia patients. NS, not significant, two-sided $t$ test, $n=36$ for each group. Data are presented as mean values $+/-\mathrm{SEM}$. Cont control, SZ schizophrenia, IN GABAergic interneurons, PN pyramidal neurons. The levels of Nrg1 mRNA were normalized to that of Gapdh. c Full-length Nrg1 type I 31 a was cloned in pMM400 between the promoter complex of TRE and CMV (cytomegalovirus minimal promoter) and SV40 polyadenylation (Poly A) signal. An HA tag was inserted between the Ig and EGF domain of NRG1. d The principle of the tet-off system. HA-Nrg1 is expressed in Gad67-tTA; TRE-Nrg1 (gtoNrg1) mice. The expression of HA-Nrg1 can be switched off by Dox. e Increased Nrg1 mRNA levels in different brain regions of gtoNrg1 mice. The Nrg1 mRNA levels return to normal after Dox treatment for 5 days. ${ }^{\star} P(P F C)=0.0157,{ }^{\star} P(S T R)=0.0223,{ }^{\star \star \star} P=0.001$, one-way-ANOVA, $n=3$ mice for each group. Data are presented as mean values +/- SEM. OB olfactory bulb, HPF hippocampus formation, STR striatum, PFC prefrontal cortex, CB cerebellum, MB midbrain. $\mathbf{f}$ Expression of HA tag in gtoNrg1 mice. Three independent experiments were repeated to get similar results. The homogenate from different brain regions was subjected to RT-PCR assay for HA and Gapdh. (1) TRE-Nrg1; (2) Gad67-tTA; (3) gtoNrg1; (4) gtoNrg1+ Dox. g Increased NRG1 protein levels (including the full length and ICD) in the PFC of gtoNrg1 mice. The homogenate of PFC from different mice was subjected to western blot and probed with anti-NRG1 and anti-GAPDH Abs. (2) control; (3) gtoNrg1; (4) gtoNrg1 + Dox. h Quantification of the expression of full-length NRG1 (top) and NRG1ICD (bottom) in panel g. ${ }^{\star} P=0.0178$ for NRG1-FL, ${ }^{\star} P=0.034$ for NRG1-ICD, one-way-ANOVA, $n=3$ mice for each group, data were normalized to controls. Data are presented as mean values $+/-$ SEM.

gtoNrg1 mice (Supplementary Fig. 3f, g). However, the startle response to $120 \mathrm{~dB}$ and PPI were compromised in gtoNrg1 mice compared with controls (Fig. 2b, c). In the prepulse + pulse trails, both genotypes reduce their startle response with increasing intensity of the prepulse (Fig. 2d). There is a minor but significant difference in the extent to which the prepulse gates the startle response to the pulse between two genotypes (interaction (prepulse $\times$ genotype) $F(2,84)=3.136, P=0.0486$, two-way ANOVA) (Fig. 2d). Together, these results suggest that overexpression of Nrg1 in GABAergic interneurons impairs startle response and has minor effects on sensorimotor gating.

Impaired social behavior is a negative symptom of schizophrenia $^{29}$. gtoNrg1 mice could distinguish the stimulus mouse and the objective in the three-chamber test, similar to controls (Supplementary Fig. 3h, i), suggesting normal social interaction. However, gtoNrg1 mice spent lesser time in the chamber with novel mice (S2) but stayed longer in the chamber with familiar mice (S1), compared with controls (Fig. 2e-g), indicating impaired social novelty. The reduced social novelty of gtoNrg1 mice may not result from the deficient olfaction because gtoNrg1 mice showed better olfaction during finding the buried food compared with controls (Supplementary Fig. 3j). We also studied nesting behavior in control and gtoNrgl mice to assess their ability to establish an organized behavior. Compared with controls, gtoNrg1 mice were unable to build an identifiable nest within $12 \mathrm{~h}$ (Fig. $2 \mathrm{~h}, \mathrm{i}$ ).
We next determined whether overexpressing Nrg1 in GABAergic interneurons affected spatial recognition memory in $\mathrm{Y}$ maze. To exclude the potential influence of hyperactivity in the Y-maze test, we analyzed the time exploring the start, old and new arms (Fig. 2j, k). Although there was a preference for the new arm in both control and gtoNrg1 mice (Fig. 2l, m), the percentage of time exploring the new arm was significantly reduced in gto $\mathrm{Nrg} 1$ mice compared with controls (Fig. 2n). These results suggest that gtoNrg1 mice have impaired spatial recognition memory.

Hypersynchrony of neural network in the PFC of gtoNrg1 mice. Given the evidence of PFC-mediated behavioral deficit such as an impaired social novelty in gto $\mathrm{Nrg} 1$ mice, we next studied how the neural network of PFC was altered in gtoNrg1 mice. Here, we focus on layers 2-3 of PFC because the layer 3 circuitry in PFC is significantly altered in schizophrenia patients ${ }^{30}$. To this end, we carried out local field potential (LFP) recordings in the PFC of freely behavioral control and gtoNrg1 mice. We acutely implanted 32-channel tetrodes in the layer 2-3 of PrL (prelimbic cortex, a major region of PFC, Fig. 3a), and after 2 weeks of recovery, the LFP was recorded. We analyzed epochs of activity in which the speed of movement was above $3 \mathrm{~cm} / \mathrm{s}$ to reduce variability in LFP recordings, and we verified that the mean speed of the epochs analyzed was similar for both genotypes. Analysis of spontaneous LFPs in layers 2-3 of PrL revealed a significant 

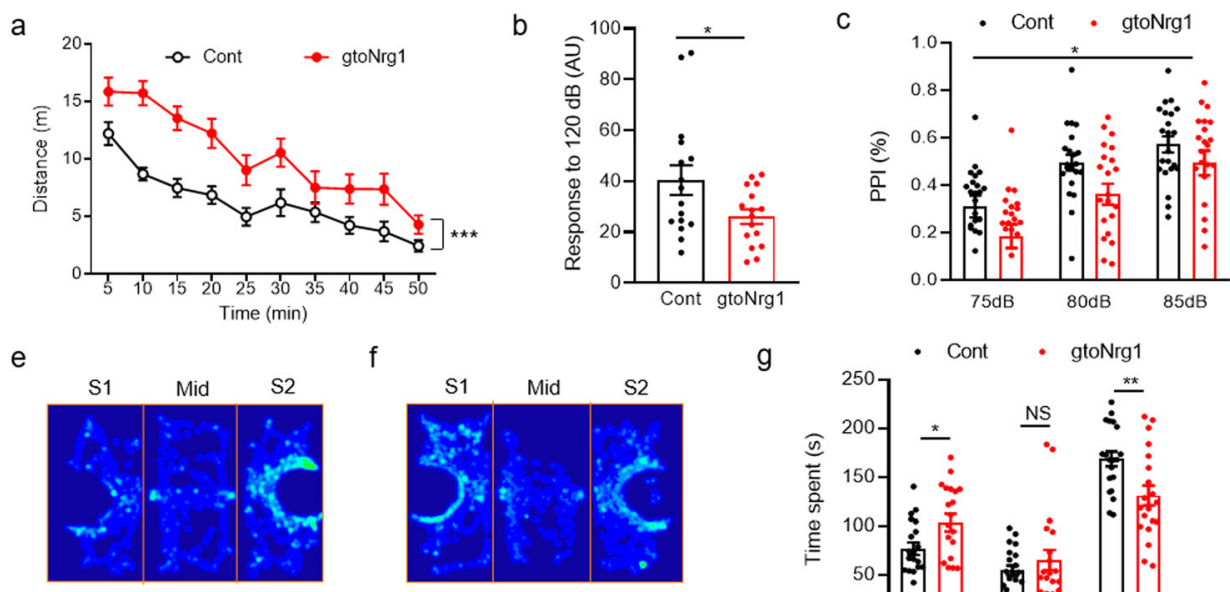

$\mathrm{f}$

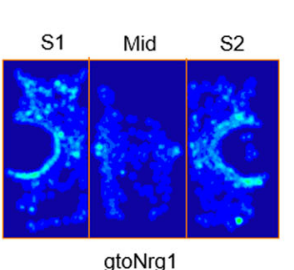

g

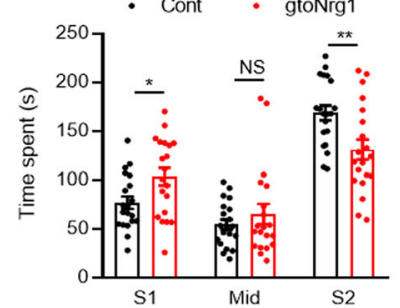

d

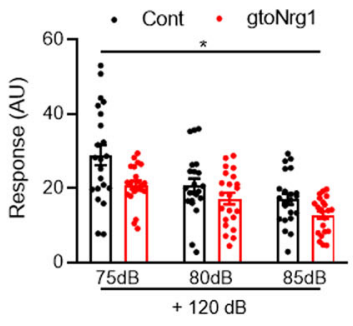

h

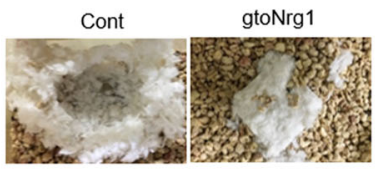

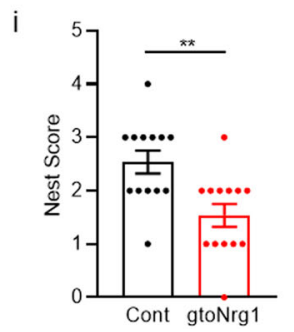

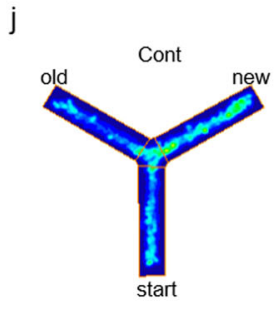

k

I

$\mathrm{m}$

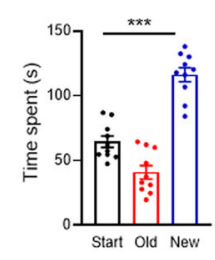

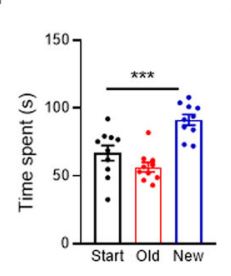

n

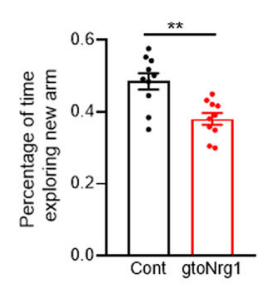

Fig. 2 Behavioral deficits in gtoNrg1 mice. a Travel distance in open field was increased in gtoNrg1 mice compared with controls. ${ }^{\star \star \star}$ Genotype $F(1,28)=$ $15.74, P=0.0005$, two-way ANOVA, $n=15$ for each group. Data are presented as mean values $+/-\mathrm{SEM}$. $\mathbf{b}$ Reduced startle response to $120 \mathrm{~dB}$ noise in gtoNrg1 mice compared with controls. ${ }^{\star} P=0.0356$, two-sided $t$ test, $n=16$ for each group. Data are presented as mean values $+/-$ SEM. $\mathbf{c}$ Impaired PPI in gtoNrg1 mice compared with controls. ${ }^{\star} G$ enotype $F(1,42)=4.9, P=0.0324$, two-way ANOVA, $n=22$ for each group. Data are presented as mean values $+/$ - SEM. d Reduced reflex amplitudes on prepulse + pulse trials in gtoNrg1 mice compared with controls. ${ }^{\star}$ Genotype $F(1,42)=6.11, P=0.0176$,

${ }^{\star \star *}$ Prepulse $F(1.709,71.78)=53.69, P<0.0001$, ${ }^{*}$ Interaction (prepulse $\times$ genotype) $F(2,84)=3.136, P=0.0486$, two-way ANOVA, $n=22$ for each group. Data are presented as mean values +/- SEM. e, f Occupancy plot of the heads from control (e) and gtoNrg1 (f) mice in the three-chamber test. S1, familiar mouse; S2, novel mouse. $\mathbf{g}$ Reduced social novelty in gtoNrg1 mice compared with controls. Time spent in each chamber was quantified. NS not significant, ${ }^{\star} P=0.02,{ }^{\star \star} P=0.0056$, two-sided $t$ test, $n=20$ for each group. Data are presented as mean values $+/-$ SEM. $\mathbf{h}, \mathbf{i}$ Impaired nest building in gtoNrg1 mice compared with controls. h Representative images for a nest built after $12 \mathrm{~h}$. $\mathbf{i}$ Quantification of nest score. ${ }^{\star \star} P=0.0031$, two-sided $t$ test, $n=13$ for each group. Data are presented as mean values + / SEM. j, $\mathbf{k}$ Occupancy plot of the heads from control (j) and gtoNrg1 (k) mice in $Y$ maze. $\mathbf{I}, \mathbf{m}$ Preference for the new arm in control (I) and gtoNrg1 ( $\mathbf{m})$ mice. The time exploring the start, old, and new arms was quantified. ${ }^{\star \star \star} P<0.001$, one-way ANOVA, $n=10$ for each group. Data are presented as mean values +/- SEM. $\mathbf{n}$ Impaired spatial recognition memory in gtoNrg1 mice compared with controls. The percentage of time exploring a new arm in the $Y$ maze was quantified. ${ }^{\star \star} P=0.0016$, two-sided $t$ test, $n=10$ for each group. Data are presented as mean values $+/-$ SEM.

increase of activity in gtoNrg1 mice, compared with controls (Fig. 3b, c). Next, we analyzed the relative power of oscillations in control and gtoNrg1 mice. We observed a significant increase in the relative power of delta $(0.5-3 \mathrm{~Hz})$, theta $(4-12 \mathrm{~Hz})$, alpha $(13-15 \mathrm{~Hz})$, and beta $(16-30 \mathrm{~Hz})$ oscillations in gtoNrg1 mice, compared with controls (Fig. $3 \mathrm{~d}-\mathrm{k}$ ). The relative power of gamma $(30-90 \mathrm{~Hz})$ and high-frequency oscillation (HFO) $(>100 \mathrm{~Hz})$ were not significantly altered in the PFC of gtoNrg1 mice, compared to controls (Fig. 3f, l-n). These results suggest that overexpression of Nrg1 in GABAergic interneurons led to hypersynchrony of the neural network in the PFC.

Elevated E/I balance in the PFC of gtoNrg1 mice. The hypersynchrony of neural networks in gtoNrg1 mice may reflect an alteration in E/I balance. Next, we sought to determine whether the $\mathrm{E} / \mathrm{I}$ ratio was changed in the PFC of gtoNrg1 mice. Toward this aim, we performed a whole-cell patch clamp to record the spontaneous excitatory and inhibitory postsynaptic currents (sEPSC and sIPSC) from layers 2 to 3 PN in PrL (Fig. 4a). As shown in Fig. $4 \mathrm{~b}-\mathrm{f}$, the frequency but not the amplitude of sEPSC was significantly increased in gtoNrg1 mice, compared with controls. By contrast, the frequency but not amplitude of sIPSC was reduced in gtoNrg1 mice, compared with controls (Fig. $4 \mathrm{~g}-\mathrm{k}$ ). In support of these results was the observation that the E/I ratio was elevated in the gtoNrg1 PFC, compared with controls (Fig. 4l). The reduced sIPSC frequency in gtoNrg1 mice (Fig. 5g, $h, j)$ might be due to the decreased GABAergic synapse number or the impaired ability of GABA release. However, these possibilities could be small because the miniature IPSC (mIPSC) (Supplementary Fig. $4 \mathrm{a}-\mathrm{c}$ ) and paired-pulse ratio (PPR) of evoked IPSC (eIPSC) (Supplementary Fig. 4d, e) from layers 2 to $3 \mathrm{PN}$ in the PrL were similar between control and gtoNrg1 mice.

We next investigate whether the reduced sIPSC frequency in gtoNrg1 mice results from the lower excitability of GABAergic interneurons. To visualize the GABAergic interneurons, we crossed Gad67-tTA and gtoNrg1 mice with TRE-Histone 2B (H2B)-GFP mice ${ }^{31}$ to get gtoGfp and gtoNrg1; Gfp mice, respectively. These mice express $\mathrm{H} 2 \mathrm{~B}-\mathrm{GFP}$ specifically in GABAergic interneurons (Supplementary Fig. 2) and were subject to whole-cell recording in PFC slices (Fig. 4m). The gtoNrg1; Gfp mice showed normal laminar structure and densities of GABAergic interneurons in PFC (Supplementary Fig. 5). The 


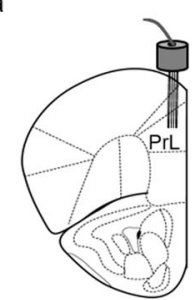

b

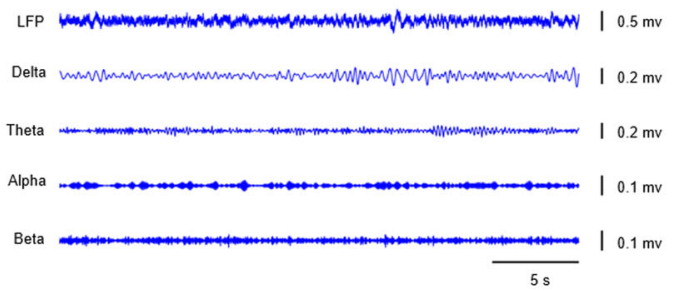

LFP

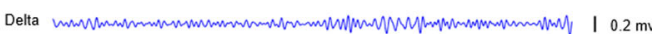
$10.2 \mathrm{mv}$ $0.1 \mathrm{mv}$

f

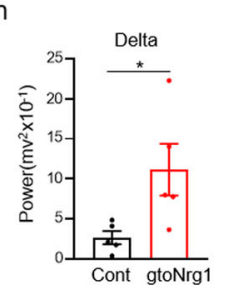

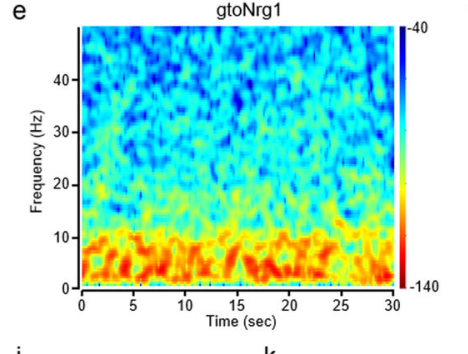
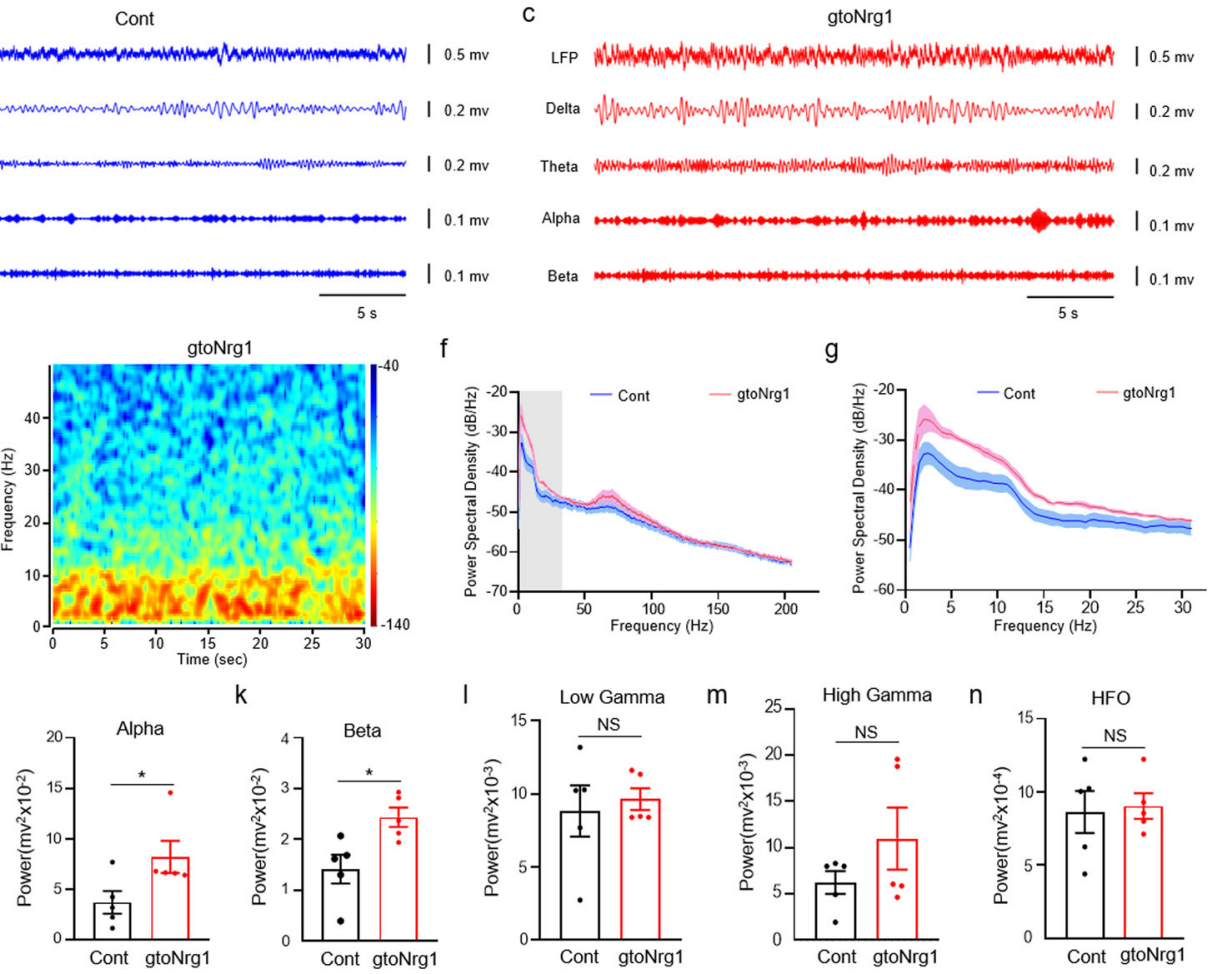

C 
a
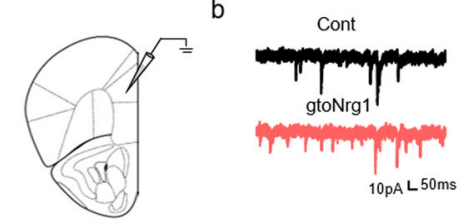

C

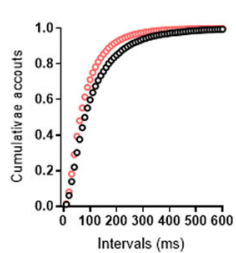

h
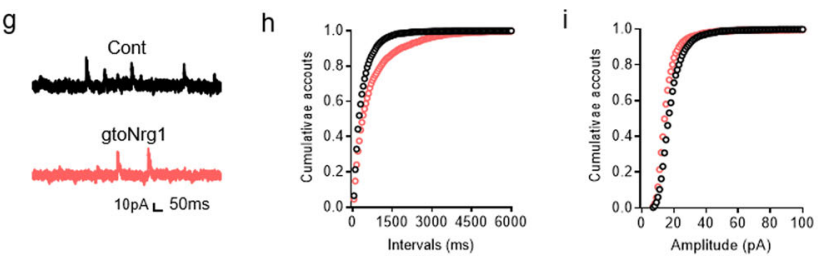

$\mathrm{m}$

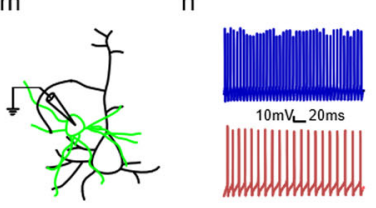

0



d

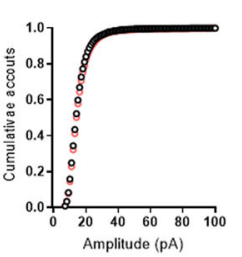

j $\quad k$

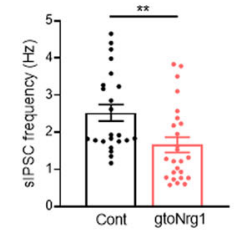

e

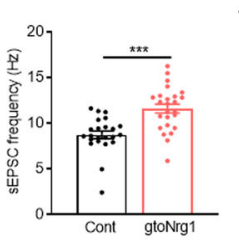



k

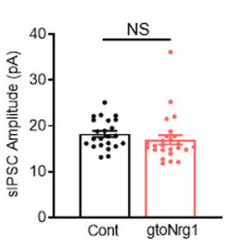

I

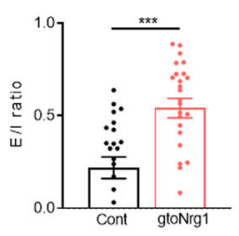



q



r



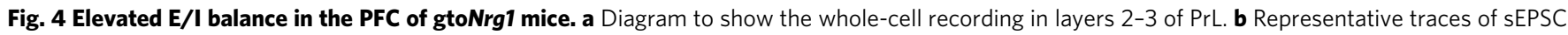
from control and gtoNrg1 mice. c, d Cumulative plots of sEPSC frequency (c) and amplitude (d). e Increased sEPSC frequency in gtoNrg1 mice. ${ }^{\star \star \star} P<$

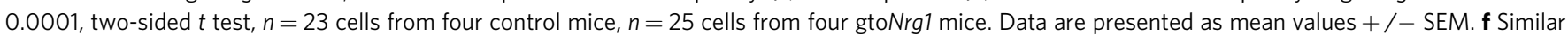

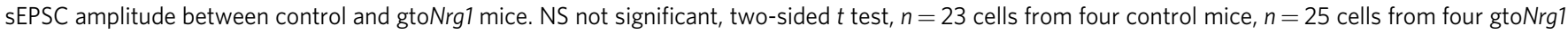
mice. Data are presented as mean values + /- SEM. $\mathbf{g}$ Representative traces of sIPSC from control and gtoNrg1 mice. h, i Cumulative plots of sIPSC frequency $(\mathbf{h})$ and amplitude (i). j Reduced sIPSC frequency in gtoNrg1 mice. ${ }^{\star \star} P=0.0068$, two-sided $t$ test, $n=23$ cells from four control mice, $n=25$ cells from four gtoNrg1 mice. Data are presented as mean values +/- SEM. k Similar sIPSC amplitude between control and gtoNrg1 mice. NS not significant, two-sided $t$ test, $n=23$ cells from four control mice, $n=25$ cells from four gtoNrg1 mice. Data are presented as mean values $+/-$ SEM. I Elevated $\mathrm{E} / \mathrm{I}$ ratio in gtoNrg1 mice. ${ }^{\star \star \star} P=0.0002$, two-sided $t$ test, $n=23$ cells from four control mice, $n=25$ cells from four gtoNrg1 mice. Data are presented as mean values +/-SEM. $\mathbf{m}$ Diagram to show the recording of GABAergic interneurons expressing EGFP. $\mathbf{n}$ Representative action potentials of FS-GABAergic interneurons. o Reduced excitability of FS-GABAergic interneurons in gtoNrg1; Gfp mice, compared with gtoGfp mice. Shown are the I/O curves of action potentials from FS-GABAergic interneurons. ${ }^{\star \star}$ Genotype $F(1,36)=8.813, P=0.0053$, two-way ANOVA, $n=17$ cells from five gtoGfp mice, $n=21$ cells from four gtoNrg1; Gfp mice. Data are presented as mean values $+/-$ SEM. p Diagram to show the recording of PN. q Representative action potentials of PN. $\mathbf{r}$ Increased excitability of PN in gtoNrg1 mice compared with controls. Shown are the I/O curves of action potentials from PN. ${ }^{\star}$ Genotype $F(1,50)=5.041, P=0.0292$, two-way ANOVA, $n=27$ cells from five control mice, $n=25$ cells from four gtoNrg1 mice. Data are presented as mean values $+/-$ SEM.

of $\mathrm{Na}_{\mathrm{v}}$ channels caused by $\mathrm{Nrgl}$ overexpression. There are four isoforms of $\mathrm{Na}_{\mathrm{v}}$ channels $\left(\mathrm{Na}_{\mathrm{v}} 1.1, \mathrm{Na}_{\mathrm{v}} 1.2, \mathrm{Na}_{\mathrm{v}} 1.3\right.$, and $\mathrm{Na}_{\mathrm{v}}$ 1.6) which are primarily expressed in the central nervous system ${ }^{33}$. The a-subunits of $\mathrm{Na}_{\mathrm{v}}$ are necessary for forming a functional ionselective channel. The $\alpha$-subunits of $\mathrm{Na}_{\mathrm{v}} 1.1, \mathrm{Na}_{\mathrm{v}} 1.2, \mathrm{Na}_{\mathrm{v}} 1.3$, and $\mathrm{Na}_{\mathrm{v}} 1.6$ are encoded by the gene Scn1a, Scn2a1, Scn3a, and Scn8a. We analyzed the transcription levels of the four $S \mathrm{cn}$ genes in the seven major neuronal clusters from the mouse frontal cortex accessible through an online database DropViz (https://dropviz. org) ${ }^{16}$ (Supplementary Fig. 6). Scn1a, Scn2a1, and Scn8a are the three major $\mathrm{Scn}$ isoforms expressed in GABAergic interneurons (Fig. 5f). The SCN1A, SCN2A1, and SCN8A proteins are similar in structure and have $74 \%$ similarity in the overall amino acid sequences $^{33}$. The expression levels of Scnla was higher in GABAergic interneurons, but lower in PN compared with Scn2a1 and Scn8a (Fig. 5f, g). These results are consistent with the previous finding that Scnla is highly expressed in PV-positive GABAergic interneurons and is critical for their excitability ${ }^{34}$. Due to these reasons, we focused on Scnla in the following study. Neither the mRNA nor the protein levels of Scnla were reduced in gtoNrg1 PFC, compared to controls (Supplementary Fig. 7a, b). The SCN1A protein levels in the membrane fraction were also similar between control and gtoNrg1 PFC (Supplementary
Fig. 7c). Together with the data in Fig. 5a-e, these results suggest that overexpression of $\mathrm{Nrgl}$ impairs the function of $\mathrm{Na}_{\mathrm{v}}$ channels rather than inhibits the gene expression or membrane trafficking of SCN1A.

Inhibition of $\mathrm{Na}_{\mathrm{v}}$ currents in GABAergic interneurons by NRG1-ICD. ErbB4 is the main receptor of NRG1 in GABAergic interneurons ${ }^{35-37}$. We next determine whether the protein levels or activity of ErbB4 were changed in gtoNrg1 mice. To this end, the homogenate of PFC from control and gtoNrg1 mice were subjected to western blots and probed with antibodies against ErbB4 and p-ErbB4. As shown in Supplementary Fig. 8, the protein levels of ErbB4 and p-ErbB4 were not altered in gtoNrg1 PFC, compared to controls. These results suggest that ErbB4 was not activated in the PFC of gtoNrg1 mice. To study whether the NRG1 EGF domain could reduce $\mathrm{Na}_{\mathrm{v}}$ currents in GABAergic interneurons, we incubated the PFC slices with 5 nM NRG1 EGF domain or BSA (as a control). NRG1 EGF domain could promote evoked GABA release (Supplementary Fig. 9a, b), which is consistent with our previous findings ${ }^{38-40}$. However, the NRG1 EGF domain did not alter the $\mathrm{Na}_{\mathrm{v}}$ currents in GABAergic interneurons (Supplementary Fig. 9c, d). 
a


j

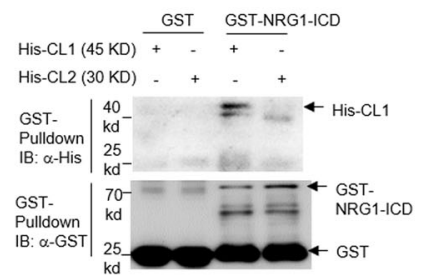

b

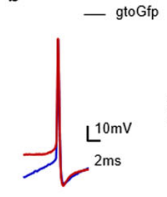

C

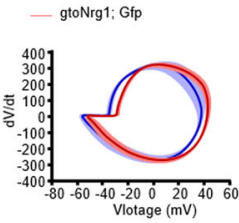

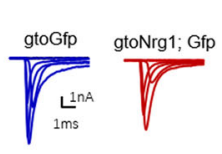

d

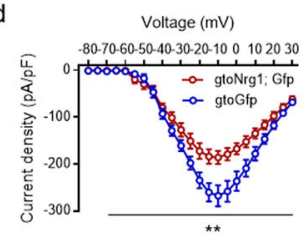

f
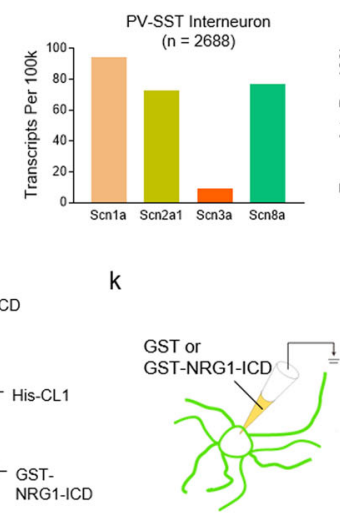

g $\mathrm{h}$



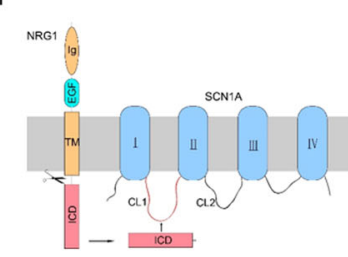

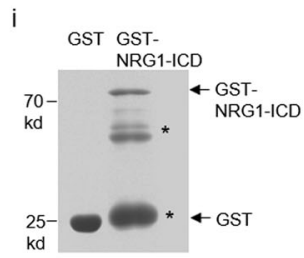

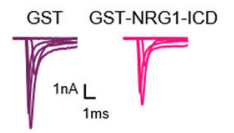

m

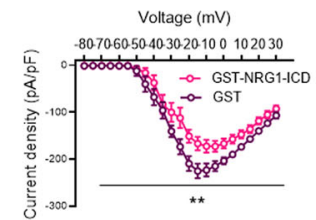

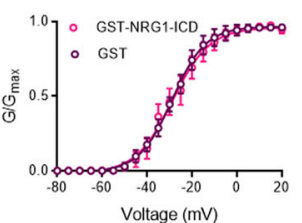

Fig. $\mathbf{5}$ Inhibition of $\mathbf{N a}_{\mathbf{v}}$ currents in GABAergic interneurons by NRG1-ICD. a Increased rheobase and depolarized action potential threshold (APT) in the FS-GABAergic interneurons of gtoNrg1; Gfp mice, compared with gtoGfp mice. ${ }^{\star} P=0.0321,{ }^{\star \star} P=0.0015$, two-sided $t$ test, $n=17$ cells from five gtoGfp mice, $n=21$ cells from four gtoNrg1; Gfp mice. $\mathbf{b}$ Representative traces of a single AP evoked from a suprathreshold current injection (left) and corresponding phase plots ( $\mathrm{dV} / \mathrm{dt}$ vs voltage) (right) recorded in FS-GABAergic interneurons from gtoGfp and gtoNrg1; Gfp mice. c Reduced Na+ current density in GABAergic interneurons from gtoNrg1; Gfp PFC. Representative current traces of Na channels in GABAergic interneurons from gtoNrg1; Gfp and gtoGfp mice. Currents were elicited by step depolarizations from -80 to $+30 \mathrm{mV}$ in $5 \mathrm{mV}$ increments from a holding potential of $-80 \mathrm{mV}$. The tracts shown are for depolarizations from -80 to $+20 \mathrm{mV}$. d I/V curves of $\mathrm{Na}_{\mathrm{v}}$ channel activation in GABAergic interneurons from gtoNrg1; Gfp and gtoGfp mice. ${ }^{\star \star}$ Genotype $F(1,28)=8.264, P=0.0076$, two-way ANOVA, $n=13$ cells from three gtoNrg1; Gfp mice, $n=17$ cells from four gtoGfp mice. Data are presented as mean values +/- SEM. e Similar voltage-dependent activation curves of $\mathrm{Na}_{\mathrm{v}}$ channels in GABAergic interneurons from gtoNrg1; Gfp and gtoGfp mice. Genotype $F(1,28)=0.023, P=0.879$, two-way ANOVA, $n=13$ cells from three gtoNrg1; Gfp mice, $n=17$ cells from four gtoGfp mice. Data are presented as mean values + /- SEM. f Transcriptional levels of Scn1a is higher than Scn2a1, Scn3a, and Scn8a in PV and SST-positive GABAergic interneurons $(n=2688$ cells). Shown are transcripts of Scn genes per $100 \mathrm{k}$ total transcripts from single-cell RNA sequencing. $\mathbf{g}$ Transcriptional levels of Scn1a were lower than Scn2a1 and Scn8a in layer 2/3 PN ( $n=41827$ cells). Shown are transcripts of Scn genes per $100 \mathrm{k}$ total transcripts from single-cell RNA sequencing. $\mathbf{h}$ Diagram showing the structure of SCN1A. The SCN1A protein was composed of four transmembrane domains (I-IV) and two major cytoplasmic loops (CL1 and CL2). The NRG1-ICD could interact with the CL1 of SCN1A. i Coomassie blue staining of $30 \mu \mathrm{g}$ GST and GST-NRG1-ICD proteins. Asterisks indicated degradation product of GST-NRG1-ICD proteins. Four independent experiments were repeated to get similar results. j Interaction of NRG1-ICD with His-CL1. The recombinant GST-NRG1-ICD and His-CL1, or His-CL2 proteins were used for GST pulldown experiments. Four independent experiments were repeated to get similar results. $\mathbf{k}$ Diagram showing delivery of GST-NRG1-ICD or GST proteins into GABAergic interneurons in gtoGfp slices. I Reduced $\mathrm{Na}^{+}$current density in GABAergic interneurons treated with GST-NRG1-ICD. Representative current traces of Na $\mathrm{v}_{\mathrm{v}}$ channels in GABAergic interneurons treated with recombinant GST or GST-NRG1-ICD proteins. Currents were elicited by step depolarizations from -80 to $+30 \mathrm{mV}$ in $5 \mathrm{mV}$ increments from a holding potential of $-80 \mathrm{mV}$. The tracts shown are for depolarizations from -80 to $+20 \mathrm{mV}$. $\mathbf{m ~ I / V ~ c u r v e s ~ o f ~ N a v ~ c h a n n e l ~}$ activation in GABAergic interneurons treated with recombinant GST or GST-NRG1-ICD proteins. ${ }^{\star \star}$ Treatment $F(1,24)=10.89, P=0.003$, two-way ANOVA, $n=14$ cells treated with GST-NRG1-ICD, $n=12$ cells treated with GST. Data are presented as mean values $+/-$ SEM. $\mathbf{n}$ Similar voltagedependent activation curves of $\mathrm{Na}_{\mathrm{v}}$ channels in GABAergic interneurons treated with GST or GST-NRG1-ICD. Genotype $F(1,24)=0.059, P=0.81$, twoway ANOVA, $n=14$ cells treated with GST-NRG1-ICD, $n=12$ cells treated with GST. Data are presented as mean values $+/-$ SEM.

Next, we study whether NRG1-ICD plays a role in modulating $\mathrm{Na}_{\mathrm{v}}$ currents in GABAergic interneurons. SCN1A contains four transmembrane domains (I-IV) and two major cytoplasmic loops (CL1 and CL2) (Fig. 5h). To investigate whether NRG1-ICD could interact with the CL of SCN1A, we purified GST-NRG1ICD, His-CL1, and His-CL2 proteins from bacteria (Fig. 5i). As a negative control, GST proteins did not bind with the His-CL1 or His-CL2 (Fig. 5j). The CL1 and CL2 are important for the neuromodulation and membrane localization of $\mathrm{SCN} 1 \mathrm{~A}$, respectively ${ }^{41}$. No interaction between NRG1-ICD and His-CL2 (Fig. 5j) implied that NRG1-ICD might not affect the membrane localization of SCN1A. In agreement, the protein levels of $\mathrm{SCN} 1 \mathrm{~A}$ in the membrane fraction were similar between control and gtoNrg1 mice (Supplementary Fig. 7c). However, NRG1-ICD could bind with His-CL1 (Fig. 5j), which suggested that NRG1ICD might modulate the function of $\mathrm{Na}_{\mathrm{v}} 1$.1channel. To further test this hypothesis, we delivered $200 \mathrm{nM}$ GST-NRG1-ICD or GST proteins (as a control) into GABAergic interneurons in gtoGfp slices through a recording pipette (Fig. 5k), and $10 \mathrm{~min}$ afterward recorded the $\mathrm{Na}_{\mathrm{v}}$ currents using voltage clamp (Fig. 5l). The I/V curves of $\mathrm{Na}_{\mathrm{v}}$ channel activation showed a downward shift in GABAergic interneurons treated with GST-NRG1-ICD, compared with control neurons (Fig. 5m). In accord, the maximal $\mathrm{Na}^{+}$current density was significantly reduced in GABAergic interneurons treated with GST-NRG1-ICD, compared with control neurons (GST-NRG1-ICD: $-172.6 \pm 11.61 \mathrm{pA} / \mathrm{pF}$, 
a

\begin{tabular}{|c|c|c|}
\hline $\begin{array}{c}\text { Mouse } \\
\text { name }\end{array}$ & Genotype & AAV treatment \\
\hline $\begin{array}{c}\text { gtoNrg1; } \\
\text { hM3Dq }\end{array}$ & $\begin{array}{c}\text { Gad67-tTA; } \\
\text { TRE-Nrg1 }\end{array}$ & $\begin{array}{c}\text { TRE-hM3Dq-P2A } \\
\text {-mCherry }\end{array}$ \\
\hline $\begin{array}{c}\text { gtoNrg1; } \\
\text { Yfp }\end{array}$ & $\begin{array}{c}\text { Gad67-tTA; } \\
\text { TRE-Nrg1 }\end{array}$ & TRE-EYFP \\
\hline
\end{tabular}
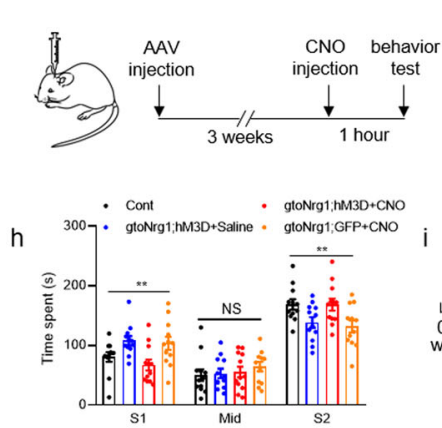

$\mathrm{m}$

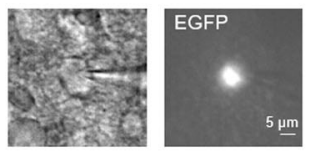

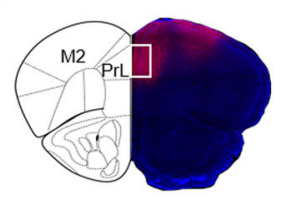

C

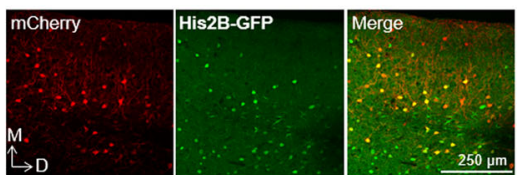

d

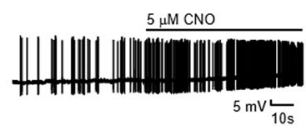

f $25+$ Cont $\rightarrow$ gtoNrg1 $\mathrm{hM} 3 \mathrm{Dq}+$ Saline

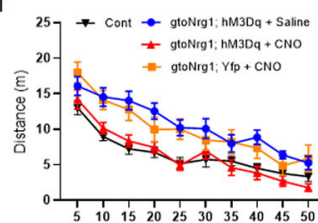

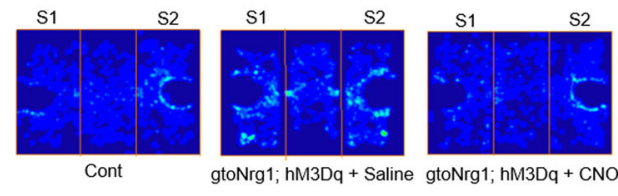

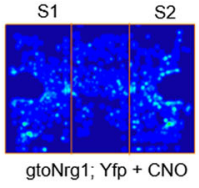

k
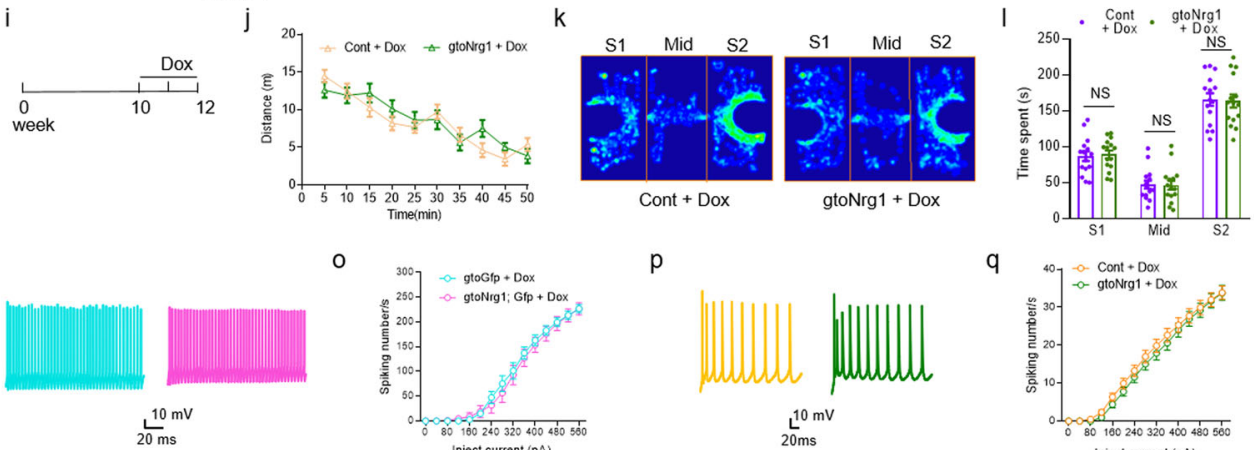

0

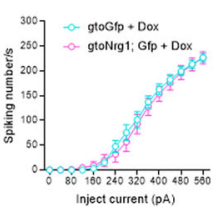

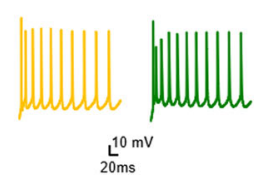

q

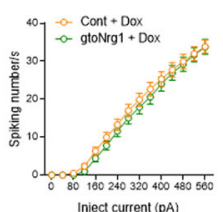

Fig. 6 Rescue of behavioral deficits and cortical disinhibition in gtoNrg1 mice. a The genotype and AAV treatment in different mouse lines. $\mathbf{b}$ Diagram to show the brain regions infected by AAV. c Expression of mCherry in GABAergic interneurons from the rectangle in panel $\mathbf{b}$. AAV expressing TRE-hM3DqP2A-mCherry were injected into the PFC of gtoGfp mice. The resulting PFC slices were subjected to immunofluorescence. Three independent experiments were repeated to get similar results. Scale bar, $250 \mu \mathrm{m}$. $\mathbf{d}$ Increased firing of FS-GABAergic interneurons by CNO in the PFC slices of gtoNrg1; Gfp mice. The AP of FS-GABAergic interneurons were recorded before and after treatment with $5 \mu \mathrm{M} C N O$. e Experimental design. Three weeks after stereotaxic injection of $A A V$, the mice were received i.p. injection of $\mathrm{CNO}(5 \mathrm{mg} / \mathrm{kg}) 1 \mathrm{~h}$ before behavioral tests. $\mathbf{f}$ Rescue of hyperactivity in gtoNrg1; hM3Dq mice by CNO. Shown is travel distance in the first 50 min during the open-field test. $F(3,50)=7.571, P=0.0003$, two-way ANOVA, $n=13$ for each group. Data are presented as mean values +/- SEM. $\mathbf{g}$ Occupancy plot of the heads from different groups of mice in the three-chamber test. S1, familiar mouse; S2, novel mouse. $\mathbf{h}$ Rescue of impaired social novelty in gtoNrg1; hM3Dq mice by CNO. Time spent in each chamber was quantified. NS not significant, ${ }^{\star \star} P=0.0068$ for $\mathrm{S} 1,{ }^{\star \star} P=0.0092$ for $\mathrm{S} 2$, one-way ANOVA, $n=12$ for each group. Data are presented as mean values $+/-\mathrm{SEM}$. $\mathbf{i}$ Experimental design . The 10-week-old control and gtoNrg1 mice were treated with Dox for 2 weeks before the behavioral test. $\mathbf{j}$ Similar travel distance between Dox-treated control and gtoNrg1 mice. Genotype $F(1,24)=0.0997, P=0.7549$, two-way ANOVA, $n=13$ for each group. Data are presented as mean values $+/-$ SEM. k Occupancy plot of the heads from Dox-treated control and gtoNrg1 mice in the three-chamber test. S1, familiar mouse; Mid, middle chamber; S2, novel mouse. I Similar social novelty between Dox-treated control and gtoNrg1 mice. Time spent in each chamber was quantified. NS, not significant, twosided $t$ test, $n=15$ for each group. Data are presented as mean values + / - SEM. $\mathbf{m}$ Expression of EGFP in the PFC slices from gtoNrg1; Gfp mice after treatment with Dox for 2 weeks. Four independent experiments were repeated to get similar results. Scale bar, $5 \mu \mathrm{m}$. $\mathbf{n}$ Representative action potential traces of FS-GABAergic interneurons in Dox-treated gtoGfp and gtoNrg1; Gfp PFC. o Similar I/O curves of action potential from FS-GABAergic interneurons in Dox-treated gtoGfp and gtoNrg1; Gfp PFC. Genotype $F(1,28)=0.094, P=0.762$, two-way ANOVA, $n=15$ cells from four mice for each group. Data are presented as mean values + / SEM. p Representative action potential traces of pyramidal neurons in Dox-treated gtoGfp and gtoNrg1; Gfp PFC. q Similar I/O curves of the action potential from pyramidal neurons in Dox-treated control and gtoNrg1 PFC. Genotype $F(1,57)=0.433, P=0.513$, two-way ANOVA, $n=29$ cells from five gtoGfp mice, $n=30$ cells from five gtoNrg1; Gfp mice. Data are presented as mean values $+/-$ SEM.

$n=14$, control: $-225.3 \pm 15.59 \mathrm{pA} / \mathrm{pF}, n=12, P=0.0109, t$ test $)$. By contrast, membrane depolarizations required for half-maximal activation of $\mathrm{Na}_{\mathrm{v}}$ channels were comparable between GABAergic interneurons treated with GST-NRG1-ICD and control neurons $\left(\mathrm{V}_{1 / 2}=-29.86 \pm 0.76 \mathrm{mV}\right.$ for GST-NRG1-ICD-treated neurons, $n=14, \mathrm{~V}_{1 / 2}=-28.76 \pm 0.69 \mathrm{mV}$ for control neurons, $n=12$, $P=0.3023, t$ test) (Fig. 5n). Together, these results indicated that acute application of NRG1-ICD into GABAergic interneurons attenuated the peak $\mathrm{Na}^{+}$current density but without changing the voltage dependence of $\mathrm{Na}_{\mathrm{v}}$ channel activation, which is similar to the results in gtoNrg1; Gfp mice (Fig. 5c-e).

The causal link between cortical disinhibition and behavioral deficits. To further demonstrate whether cortical disinhibition in PFC causes behavioral deficits in gto $\mathrm{Nrg} 1$ mice, we performed rescue experiments using chemogenetic approaches. Toward this goal, we bilaterally injected adeno-associated virus (AAV) expressing TRE-hM3Dq-P2A-mCherry, an excitatory DREADD (designer receptors exclusively activated by designer drugs ${ }^{42}$ ) or TRE-EYFP (as a control) into PFC of gtoNrg1 mice. The resulting gtoNrg1 mice expressing TRE-hM3Dq-P2A-mCherry or TREEYFP were named gtoNrg1; hM3Dq or gtoNrg1; Yfp mice, respectively (Fig. 6a). The brain regions infected by AAV were mainly PrL and M2 motor cortex (Fig. 6b). Since the expression of $\mathrm{hM} 3 \mathrm{Dq}$ is controlled by TRE promoter and $\mathrm{tTA}, \mathrm{hM} 3 \mathrm{Dq}$ is only expressed in GABAergic interneurons from gtoNrg1; hM3Dq mice (Fig. 6c). Since we used the excitatory DREADD, administration of $\mathrm{CNO}$ increased the excitability of FSGABAergic interneurons in gtoNrg1; hM3Dq mice (Fig. 6d).

Three weeks after AAV administration, mice were i.p. injected with $\mathrm{CNO}$ or saline $1 \mathrm{~h}$ prior to the behavioral test (Fig. 6e). 
When gtoNrg1; hM3Dq mice were treated with saline, they showed hyperactivity compared with controls (Fig. 6f). However, they showed normal locomotion after treatment with $\mathrm{CNO}$ (Fig. 6f). The effects of $\mathrm{CNO}$ are specific because it cannot rescue hyperactivity in gtoNrg1; Yfp mice (Fig. 6f). These results indicate that activation of GABAergic interneurons in the PFC could rescue hyperactivity in gtoNrg1 mice.

When gtoNrg1; hM3Dq mice were treated with saline, they showed impairment in social novelty compared with controls (Fig. $6 \mathrm{~g}, \mathrm{~h}$ ). However, the social novelty became normal in gtoNrg1; hM3Dq mice after treatment with CNO (Fig. 6g, h). The effects of $\mathrm{CNO}$ are specific because it cannot reverse impaired social novelty in gtoNrg1; Yfp mice (Fig. 6g, h). These results indicated that activation of GABAergic interneurons in the PFC could reverse the social behavioral deficit in gtoNrg1 mice. Altogether, these data demonstrate a causal link between PFC disinhibition and behavioral deficits in gtoNrg1 mice.

Dependence of behavioral deficits and cortical disinhibition on continuous NRG1 overexpression. Since Gad67-tTA starts to be expressed from embryonic stage ${ }^{27}$, the phenotypes seen in gtoNrg1 mice could be due to overexpressing NRG1 during development or the persistent NRG1 increase in adulthood. The rescue experiments using chemogenetic approaches suggested that the behavioral deficits in gtoNrg1 mice might be reversible. To further test this hypothesis, adult mice at 2.5-month-old were fed with Dox-containing water for 2 weeks (Fig. 6i). To eliminate possible compounding effects of Dox, both control and gtoNrg1 mice were subjected to Dox treatment. Notably, Dox-treated gtoNrg1 and control mice traveled a similar distance in the open field (Fig. 6j), suggesting normal locomotive activity in Doxtreated gtoNrg1 mice. In addition, Dox-treated gtoNrg1 and control mice showed similar performance in the three-chamber test (Fig. 6k, l), indicating normal social novelty in Dox-treated gtoNrg1 mice.

If the cortical disinhibition is a contributing mechanism, it should be diminished when NRG1 expression returned to normal levels. To test this notion, Dox-treated mice were subjected to electrophysiological recordings. Please note that EGFP is still visible in the gtoNrg1; Gfp PFC slices after treatment with Dox for 2 weeks (Fig. $6 \mathrm{~m}$ ) due to the long half-life of EGFP. These observations enable us to visualize GABAergic interneurons in the gtoNrg1; Gfp PFC slices after turning off NRG1 overexpression. The I/O curve of AP from FS-GABAergic interneurons was similar between Dox-treated gtoGfp and gtoNrg1; Gfp mice (Fig. 6n, o), indicative of normal excitability. Likewise, the enhanced firing rate of PN in gtoNrg1 PFC also returned to control levels after Dox treatment (Fig. 6p, q). Altogether, these results demonstrate that behavioral deficits and cortical disinhibition in gtoNrg1 mice require continuous NRG1 overexpression.

\section{Discussion}

In this study, we first analyzed Nrg1 gene expression in the postmortem PFC and found increased type I Nrg1 expression in GABAergic interneurons from schizophrenia patients, compared with age- and sex-matched controls. Then we generated gto $\mathrm{Nrg} 1$ mice where type I Nrg1 was specifically overexpressed in GABAergic interneurons. Intriguingly, gtoNrg1 mice exhibited cortical disinhibition at the cellular, synaptic, and neural network levels. We further demonstrated that cortical disinhibition led to behavioral deficits. Lastly, we illustrated that both cortical disinhibition and behavioral deficits in gtoNrg1 mice depended on continuous NRG1 overexpressing. In sum, our results demonstrate mechanisms underlying cortical disinhibition related to schizophrenia and raise a possibility that relevant brain disorders may benefit from intervention to restore NRG1 signaling and GABAergic function.

Chemogenetic activation of GABAergic interneurons may increase tonic inhibition, but cannot restore the temporal organization of inhibitory transmission. Therefore, the rescue of locomotor and social novelty behavior by the chemogenetic manipulation suggests that these behaviors depend on tonic prefrontal inhibition, but do not require precise temporal regulation of this inhibition ${ }^{43,44}$. In line with this notion, the schizophrenia-relevant behavioral deficit such as hyperactivity was also found in other pharmacological models of disinhibition in the forebrain regions ${ }^{45-48}$.

Recent studies from single-cell RNA sequencing revealed that Nrg1 is expressed in both glutamatergic and GABAergic neurons in mouse and human $\mathrm{PFC}^{16,17}$. Previous studies mainly focused on the function of NRG1 in glutamatergic pyramidal neurons ${ }^{49-52}$. Overexpression of $\mathrm{Nrgl}$ in pyramidal neurons led to glutamatergic hypofunction through inhibiting glutamate release or impairing NMDA receptor ${ }^{26,53-55}$. This study showed that the excitability of FS-GABAergic interneurons was impaired in gtoNrg1 mice where Nrg1 was overexpressed in GABAergic interneurons. By contrast, the excitability of FS-GABAergic interneuron was not reduced in ctoNrg1 mice where $\mathrm{Nrg1}$ was overexpressed in excitatory pyramidal neurons ${ }^{26}$. Recent studies indicated that NRG1 was highly expressed in PV-positive GABAergic interneurons and was important for the plasticity of the visual cortex ${ }^{56,57}$. Together, these results demonstrated the function of NRG1 in GABAergic interneurons.

gtoNrg1 mice exhibited an increased theta and delta oscillations in the PFC under freely behavioral conditions. By contrast, a previous study showed that overexpression of NRG1 in pyramidal neurons enhanced gamma oscillations in hippocampal slices ${ }^{50}$. The discrepancy between these two studies might result from different neuronal types where NRG1 is overexpressed or the different conditions from in vivo and in vitro. Increased theta and delta oscillations are two of the consistent observations in schizophrenia patients ${ }^{58}$. By contrast, both increased and reduced gamma oscillation have been reported in schizophrenia patients under different conditions ${ }^{59}$.

The NRG1 EGF domain acts on the ErbB4 receptor which is mainly expressed in GABAergic interneuron ${ }^{35-37}$. NRG1-ErbB4 signaling has been implicated in GABAergic circuity formation $^{35,60-63}$ and GABA transmission $38-40,64,65$. However, ErbB4 was not activated in the PFC of gtoNrg1 mice. The NRG1 protein levels in gtoNrg1 PFC were 1.5-2-folds higher than controls and were much lower than other $\mathrm{Nrgl}$ transgenic mouse lines ${ }^{50,53}$, which might explain why ErbB4 was not activated in gtoNrg1 PFC. We further showed that the NRG1 EGF domain did not cause the reduction of $\mathrm{Na}_{\mathrm{v}}$ currents in GABAergic interneurons. Indeed, the NRG1 EGF domain has been shown to increase the excitability of GABAergic interneurons through $\mathrm{K}^{+}$ channels $s^{66}$, albeit an opposite effect was observed in cultured hippocampal neurons ${ }^{67}$.

On the other hand, NRG1-ICD can induce downstream signaling through protein-protein interaction and gene transcription $7,26,68-70$. The reduced excitability of FS-GABAergic interneurons in gtoNrg1 mice might be due to the effects of NRG1-ICD. In support of this hypothesis was the observation that NRG1-ICD could interact with the cytoplasmic loop 1 of SCN1A and inhibit the peak $\mathrm{Na}_{\mathrm{v}}$ currents. The cytoplasmic loop 1 of SCN1A has been shown to interact with signaling proteins or be phosphorylated by protein kinases, which leads to the reduction of the peak $\mathrm{Na}_{\mathrm{v}}$ currents ${ }^{41,71}$. We speculate that the interaction of NRG1-ICD with the cytoplasmic loop 1 of SCN1A might account for the inhibition of peak $\mathrm{Na}_{\mathrm{v}}$ currents by 
NRG1-ICD. Given that NRG1-ICD but not NRG1 EGF domain attenuated peak $\mathrm{Na}_{\mathrm{v}}$ currents in GABAergic interneurons, a parsimonious explanation of our finding is that the reduction of peak $\mathrm{Na}_{\mathrm{v}}$ currents in gtoNrg1 mice is due to NRG1-ICD. In sum, the data presented here demonstrate mechanisms underlying how Nrg1 dysregulation impairs brain function, which might provide insight into the pathophysiological mechanisms of schizophrenia.

\section{Methods}

Generation of gtoNrg1 mice. The TRE-Nrg1 mice were generated as described in our paper ${ }^{26}$. Briefly, Nrg1I $\beta 1$ a was cloned into the EcoR V site of pMM400. An HA tag was inserted between Ig and EGF domains. A Not I fragment containing the transgene was used for transgenic mouse production. Gad67-tTA mice ${ }^{27}$ were kindly provided by Dr. Yuchio Yanagawa, Gunma University, Japan. TRE-H2BGFP reporter mice were from Jackson laboratory (005104). The TRE-Nrg1 transgene mice and heterozygous Gad67-tTA knock-in mice were backcrossed with C57BL/6 mice for more than ten generations before cross-breeding. The resulting offspring contain four genotypes: wt, Gad67-tTA, TRE-Nrg1, and gtoNrg1. The Gad67-tTA heterozygous knock-in mice have an insertion of tTA cassette after the start codon of Gad67 gene ${ }^{27}$, which might disrupt Gad67 gene expression. To avoid the potential knock-in effect on Gad67 gene expression, we used Gad67-tTA mice as littermate controls for gtoNrg1 mice. To eliminate the possible effects of the hormone cycle, male mice were used in all experiments. Animals were housed in rooms at $23{ }^{\circ} \mathrm{C}$ and $50 \%$ humidity in a $12 \mathrm{~h}$ light/dark cycle and with food and water available ad libitum. In some experiments, Dox (Sigma-Aldrich, catalog number D9891) was added to drinking water at $1 \mathrm{mg} / \mathrm{ml}$ in $2.5 \%$ sucrose. Animal experimental procedures were approved by the Institutional Animal Care and Use Committee of East China Normal University.

RT-qPCR and single-cell RT-PCR. The total RNA was isolated from the mouse brain and purified using Triazol (Invitrogen) and an RNAeasy mini kit (Qiagen), respectively. In total, $5 \mu \mathrm{g}$ of total RNA was reverse transcribed using oligoT primers and SuperScript III reverse transcriptase (Invitrogen). One percent of the resulting cDNA was analyzed by qPCR in triplicates using SYBR Green/ROX (Fermentas) on Chromo 4 (Bio-Rad). The following primer pairs were used: type 1 Nrg1-F: gagtcagctgcaggctccaagc; type 1 Nrg1-R: gtgatgttggca gaggcactgtc; Gapdh-F: gtggagtcatactggaacatgtag; Gapdh-R: aatggtgaaggtcggtgtg. Levels of target mRNA levels were normalized to levels of Gapdh mRNA measured at the same time on the same reaction plate.

The PFC slices from gtoNrg1; Gfp mice were used for single-cell RT-PCR. The neurons expressing EGFP were GABAergic interneurons while the neurons with a triangle shape that do not express EGFP were excitatory pyramidal neurons. After a cell is patched, the intracellular contents are aspirated into the patch pipette and used for RT-PCR. Strict RNase-free solutions and pipettes were used for collecting single-cell RNA samples. We performed single neuron RT-PCR following the protocol in the paper ${ }^{72}$. The following primer pairs were used: HA-F: tgggaccagcatcgattacccat; HA-R: cgaccaccagcagggcgatac; vGat-F: tcacgacaaacccaagatcac; vGat-R: gtcttcgttctcc tcgtacag; vGlut1-F: cacagaaagcccagttcaac; vGlut1-R: catgtttagggtggaggtagc.

\section{Immunofluorescence. The process of immunofluorescence analysis was per-} formed as described by our previous studies ${ }^{26}$. Briefly, brain slices were permeabilized with $0.3 \%$ Triton-X 100 and 5\% BSA in PBS and incubated with primary antibodies at $4{ }^{\circ} \mathrm{C}$ overnight. After washing with PBS three times, samples were incubated with Alexa Fluor-555 secondary antibodies (goat-anti-mouse, A32727; goat-anti-rabbit, A32732; 1:1000, Thermo Fisher) for $1 \mathrm{~h}$ at room temperature. Samples were mounted with Vectashield mounting medium (Vector Labs) and images were taken by Leica TCS SP8 confocal microscope. The following primary antibodies were used: rabbit anti-NeuN (1:500, Abcam, ab177487), mouse antiGABA (1:1000, Invitrogen, PA5-32241), and rabbit anti-neurogranin (1:1000, R\&D, MAB7947). Unbiased stereology TissueFAX Plus ST (Tissue Gnostics, Vienna, Austria) $)^{73}$ was applied to count EGFP-positive and NeuN-positive cell number in brain slices.

Western blot. Homogenates of PFC were prepared in RIPA buffer containing $50 \mathrm{mM}$ Tris- $\mathrm{HCl}, \mathrm{pH} 7.4,150 \mathrm{mM} \mathrm{NaCl}, 2 \mathrm{mM}$ EDTA, $1 \%$ sodium deoxycholate, $1 \%$ SDS, $1 \mathrm{mM}$ PMSF, $50 \mathrm{mM}$ sodium fluoride, $1 \mathrm{mM}$ sodium vanadate, $1 \mathrm{mM}$ DTT, and protease inhibitors cocktails. The supernatant of homogenate was subjected to centrifugation $(25,000 \times g \times 1 \mathrm{~h})$ to obtain the membrane fraction which was dissolved by RIPA buffer containing $1 \mathrm{M}$ urea. All the protein samples were boiled in $100^{\circ} \mathrm{C}$ water bath for $10 \mathrm{~min}$ before western blot. Homogenates or membrane protein were resolved on SDS/PAGE and transferred to nitrocellulose membranes, which were incubated in the TBS buffer containing $0.1 \%$ Tween-20 and $5 \%$ milk for $1 \mathrm{~h}$ at room temperature before the addition of primary antibody for incubation overnight at $4{ }^{\circ} \mathrm{C}$. After wash, the membranes were incubated with HRP-conjugated secondary antibody (goat-anti-mouse, G-21040; goat-anti-rabbit, G-21234; 1:2000, Thermo Fisher) in the same TBS buffer for $1 \mathrm{~h}$ at room temperature. Immunoreactive bands were visualized by ChemiDocTM XRS + Imaging System (BIO-RAD) using enhanced chemiluminescence (Pierce) and analyzed with Image J (NIH). The following antibodies were used: rabbit antiNRG1 (1:1000, Santa Cruz, sc-348), mouse anti-GAPDH (1:5000, Abways, ab0037), mouse anti-PSD95 (1:1000, Millipore, 2492127), rabbit anti-ErbB4 (1:1000, Cell Signaling, 111B2), rabbit anti-p-ErbB4 (1:200, Cell Signaling, Y1248) and rabbit anti- $\mathrm{Na}_{\mathrm{v}} 1.1$ (1:500, Alomone Labs, ASC-001), mouse anti-GST (1:5000, ImmunoWay, B2101) and mouse anti-His (1:2000, ImmunoWay, B0401).

Behavior analysis. To eliminate possible effects that may be associated with the knock-in into Gad67 gene, Gad67-tTA mice were used as controls in behavioral analysis. Behavioral tests were performed in 2.5-3-months old, male mice. The investigators for behavioral tests were blind to genotypes and/or Dox administration. Both control and gtoNrgl mice were treated with Dox to avoid possible compounding effects of Dox on behaviors. The behaviors were tested in the following order: open field, social interaction, social novelty, Y maze, and PPI. The intertest intervals are 2-3 days, except that between social interaction and novelty ( $30 \mathrm{~min}$ ). The second batch of mice was used for nest building and buried foodfinding test. The third batch of mice was used for behavioral tests after treatment with Dox. Mice were not tested for the same behavioral paradigms more than once, to avoid the effects of learning and memory.

Open field. Mice were placed in a chamber $(27.9 \times 27.9 \times 20.3 \mathrm{~cm})$ and monitored for movement for 50 min using an infrared camera placed above the box. The total distance, time in the center and margin of open field was measured by ANY-maze video tracking system (Stoelting).

Prepulse inhibition (PPI). PPI tests were conducted in the SR-LAB TM Startle Response System (San Diego Instruments). The motion of mice, placed in a Plexiglas tube mounted on a plastic frame, was monitored by a piezoelectric accelerometer. Before the test, mice were allowed to habituate to the chamber, to the $70-\mathrm{dB}$ background white noise for $5 \mathrm{~min}$, and to the prepulse $(20 \mathrm{~ms}$ white noise at 75,80 , or $85 \mathrm{~dB})$ and auditory-evoked startle stimuli $(120 \mathrm{~dB}, 20 \mathrm{~ms})$. In the PPI test, mice were subjected to 12 startle trials $(120 \mathrm{~dB}, 20 \mathrm{~ms})$ and 12 prepulse/startle trials $(20 \mathrm{~ms}$ white noise at 75,80 , or $85 \mathrm{~dB}$ at $100-\mathrm{ms}$ intervals and 20 ms $120-\mathrm{dB}$ startle stimulus). Different trial types were presented pseudo-randomly with each trial type presented 12 times, and no two consecutive trials were identical. Mouse movement was measured for $100 \mathrm{~ms}$ after the startle stimulus onset (sampling frequency $1 \mathrm{kHz}$ ) for $100 \mathrm{~ms}$. PPI (\%) was calculated according to the formula: (100 - (startle amplitude on prepulse-pulse trials/startle amplitude on pulse alone trials) $\times 100$ )

Social interaction and novelty. Adult male mice were tested for social behavior in a three-chamber box $(60 \times 40 \times 25 \mathrm{~cm})$. Each of the end chambers contains a clear Plexiglas cylinder. One cylinder is the "social" cylinder, which contains a stimulus mouse (adult wild-type male mice who never met test mice). The other cylinder is "non-social" cylinder, which is empty. The test mice were first placed in the center chamber and allowed to freely explore the chambers with two empty cylinders for $10 \mathrm{~min}$. For the social interaction test, mice were given an additional $5 \mathrm{~min}$ to explore the chambers with a "social" cylinder (S1) and a "non-social" cylinder (O). Thirty min after the social interaction test, the mice were subjected to a social novelty test. The test mice were given an additional $5 \mathrm{~min}$ to explore the chambers with cylinders containing a familiar mouse (S1) and a novel mouse (S2). Six-weekold male mice on a C57BL/6 background were used as social opponents. Sessions were video-recorded, and time spent around the "social" cylinder and "non-social" cylinder, or the familiar cylinder and novel cylinder were analyzed by the ANYmaze video tracking system (Stoelting). The box and cylinder were cleaned with $75 \%$ ethanol and dried thoroughly after each test session.

$Y$ maze. The Y-maze apparatus was shaped like a $\mathrm{Y}$ and had three identical arms $(25 \times 10 \times 10 \mathrm{~cm})$ placed at an angle of $120^{\circ}$ with respect to each other. The three arms were respectively labeled A (start arm), B (old arm), and C (new arm). There are different visual cues on the wall at the end of each arm. Mice were placed at the end of one arm (A) and allowed to freely navigate A and B arm for 5 min while C arm is blocked. Ten minutes later, $\mathrm{C}$ arm is opened, and the mice were allowed to freely explore all three arms for an additional $4 \mathrm{~min}$. When the limbs of mouse were positioned in the arm was considered to have entered an arm. The activity of the mice was recorded and analyzed by the ANY-maze video tracking system (Stoelting). The apparatus was cleaned with $75 \%$ ethanol and dried thoroughly after each test session.

Nest building. One cotton square $(5 \times 5 \mathrm{~cm})$ was placed in one cage with a single mouse. Twelve hours later, the nest was evaluated by a 5 -point nest-rating scale ${ }^{74}$ Each nest was evaluated by six independent investigators who are blinded to the genotype of the mice. The score of each nest was averaged by that from six investigators.

Buried food-finding test. The mice were subject to food deprivation $18 \mathrm{~h}$ before test. The test begins by placing a mouse in a clean cage $(36 \mathrm{~cm} \mathrm{~L} \times 20 \mathrm{~cm} \mathrm{~W} \times 18 \mathrm{~cm} \mathrm{H})$ 
containing $3 \mathrm{~cm}$ deep of clean bedding. The subject is allowed to acclimate to the cage for $5 \mathrm{~min}$. Then the mouse was transferred to an empty clean cage. We buried $2 \mathrm{~g}$ food pellet $\sim 1 \mathrm{~cm}$ beneath the surface, in a random corner of the cage. Smooth out the surface and reintroduce the mouse to the cage. The mouse is considered to have uncovered the food when it starts to eat, usually holding the food with forepaws. The latency to find the food was recorded by the investigator.

Recording in freely moving mice. The microdrive containing eight tetrodes (each tetrode has four channels) was prepared as described in our previous study ${ }^{75}$. The mouse was anesthetized with pentobarbital sodium $(40 \mathrm{mg} / \mathrm{kg})$ before implanting the microdrive into PrL with the coordinates: anteroposterior (AP) $1.9 \mathrm{~mm}$, mediolateral (ML) $0.5 \mathrm{~mm}$ relative to bregma. The tips of tetrodes were advanced to $1.4 \mathrm{~mm}$ from pia in the depth. After surgery, animals were housed in home cages to recover for 2 weeks. We used Plexon MAP system (Plexon, USA) to monitor neuronal signals. The tetrodes advanced $35 \mu \mathrm{m}$ every other day via rotating the screw. When the tetrodes reached layers 2-3 of PrL, the recording was started. The mouse behavior was monitored simultaneously by a video. The awake active state (speed $>3 \mathrm{~cm} / \mathrm{s}$ ) was determined by the video and real-time spectrum. The power of LFP at a different frequency (delta: $1-3 \mathrm{~Hz}$, theta: $4-12 \mathrm{~Hz}$, alpha: $13-15 \mathrm{~Hz}$, beta: 16-30 Hz, low gamma: $30-50 \mathrm{~Hz}$, high gamma: $55-90 \mathrm{~Hz}$, HFO: $100-300 \mathrm{~Hz}$ ) was analyzed through Welch's averaged periodogram with a 1024-ms nonoverlapping Hanning window $(\mathrm{NFFT}=2048)$ in combination with the function of PWELCH in Matlab R2013a.

Electrophysiology. TRE-Nrg1 and Gad67-tTA mice showed no difference in electrophysiological studies, compared to wild-type mice. Gad67-tTA mice were used as controls in all electrophysiological studies. Mice were anesthetized by ketamine/xylazine (Sigma) and perfused transcardially for $1 \mathrm{~min}$ with $4^{\circ} \mathrm{C}$ modified artificial cerebrospinal fluid (aCSF) containing (in mM) 250 glycerol, $2 \mathrm{KCl}$, $10 \mathrm{MgSO}_{4}, 0.2 \mathrm{CaCl}_{2}, 1.3 \mathrm{NaH}_{2} \mathrm{PO}_{4}, 26 \mathrm{NaHCO}_{3}$, and 10 glucose, to protect CNS neurons and maintain functional connectivity of brain slices. Mice were then decapitated and brains were quickly removed and chilled in ice-cold ACSF for an additional $1 \mathrm{~min}$. Transverse mPFC slices $(350 \mu \mathrm{m})$ were prepared using a Vibroslice (VT 1000 S; Leica) in ice-cold ACSF. Slices were then incubated in regular ASCF containing (in mM): $126 \mathrm{NaCl}, 3 \mathrm{KCl}, 1.25 \mathrm{NaH}_{2} \mathrm{PO}_{4}, 1.0 \mathrm{MgSO}_{4}, 2.0$ $\mathrm{CaCl}_{2}, 26 \mathrm{NaHCO}_{3}$, and 10 glucose for $30 \mathrm{~min}$ at $34^{\circ} \mathrm{C}$ for recovery, and then at room temperature $\left(25 \pm 1{ }^{\circ} \mathrm{C}\right)$ for an additional $2-8 \mathrm{~h}$. All solutions were saturated with $95 \% \mathrm{O}_{2} / 5 \% \mathrm{CO}_{2}$ ( $\left.\mathrm{vol} / \mathrm{vol}\right)$. Dox $(10 \mathrm{ng} / \mathrm{ml})$ was present in the perfusate for experiments with slices from Dox-treated mice.

Whole-cell patch-clamp recordings from layers 2 to $3 \mathrm{PN}$ in PrL were visualized with infrared optics using an upright microscope equipped with a $\times 40$ water-immersion lens (BX51WI; Olympus) and infrared-sensitive CCD camera. All data were obtained with a HEKA EPC10 double patch-clamp amplifier. Data were low-pass filtered at $10 \mathrm{kHz}$ and digitally sampled at $10 \mathrm{kHz}$ with PatchMaster version $2 \times 90.1$. To record sEPSC and sIPSC, the pipettes were filled with the solution (in $\mathrm{mM}$ ): $135 \mathrm{CsCH}_{3} \mathrm{SO}_{3}, 5 \mathrm{CsCl}, 5 \mathrm{TEA}-\mathrm{Cl}, 20 \mathrm{HEPES}$, 0.4 EGTA, 2.5 Mg-ATP, $0.25 \mathrm{Na}-\mathrm{GTP}$, and 1 QX314 (pH 7.25, $290 \mathrm{mOsm}$ ). Membrane potential was held at $-70 \mathrm{mV}$ for sEPSCs and $0 \mathrm{mV}$ for sIPSCs, respectively. To record eIPSCs, the pipettes were filled with the solution (in $\mathrm{mM}$ ): $130 \mathrm{CsCH}_{3} \mathrm{SO}_{3}, 10 \mathrm{CsCl}, 10$ HEPES, 0.2 EGTA, $1 \mathrm{MgCl}_{2}, 4 \mathrm{Mg}$-ATP, $0.3 \mathrm{Na}-\mathrm{GTP}$, and $5 \mathrm{QX} 314$ ( $\mathrm{pH} 7.25,285 \mathrm{mOsm})$. The neurons were holding at $-70 \mathrm{mV}$ which were stimulated with a $100 \mu \mathrm{s}$ current injection by a nichromewire electrode placed 50-100 $\mu \mathrm{m}$ from the soma of recorded neurons. To record mIPSCs, the concentration of $\mathrm{CsCl}$ was increased to $140 \mathrm{mM}, \mathrm{CsCH}_{3} \mathrm{SO}_{3}$ was omitted to enhance the driving force of $\mathrm{Cl}^{-}$, and $1 \mu \mathrm{M}$ TTX was added in the bath solution. To record $\mathrm{Na}_{\mathrm{v}}$ currents, $\mathrm{CdCl}_{2}(120 \mu \mathrm{M})$ and $\mathrm{CNQX}(20 \mu \mathrm{M})$ were added to the aCSF to block $\mathrm{Ca}^{2+}$ and AMPA receptor currents. The $\mathrm{Na}_{\mathrm{v}}$ currents were evoked with a series of $100 \mathrm{~ms}$ depolarizations from a holding potential of $-80 \mathrm{mV}$ to $+30 \mathrm{mV}$ in $5 \mathrm{mV}$ increments. Activation curves of $\mathrm{Na}_{\mathrm{v}}$ channels were fitted to Boltzmann relationships. In all protocols, the intersweep interval was 2 s. Spontaneous and miniature events were analyzed using Mini Analysis Program (Synaptosoft). E/I ratio was calculated by (charge $e^{\mathrm{sESC}}-$ charge $\left.^{\mathrm{sIPSC}}\right) /$ (charge ${ }^{\mathrm{SEPSC}}+$ charge $^{\mathrm{sIPSC}}$ ), where charge ${ }^{\mathrm{sEPSC}}$ and charge ${ }^{\mathrm{sIPSC}}$ represent total charge of sEPSC and SIPSC, respectively.

The action potential was recorded by the current-clamp. Neurons were held at $-80 \mathrm{mV}$ and were injected with different currents (duration, $500 \mathrm{~ms}$; increments, $\pm 20 \mathrm{pA}$; from -200 to $580 \mathrm{pA}$; interval, $10 \mathrm{~s}$ ). The input-output relationship was defined as the number of action potentials versus the amplitude of current injection. Input resistance was determined as the slope of the linear regression of the I-V plot for a series of hyperpolarizing pulses, where I is current amplitude and $\mathrm{V}$ is the steady-state voltage. The basic electrophysiological characteristics were measured for the first action potential waveform during the depolarization. The action potential threshold was calculated as the voltage corresponding to the peak of the third differential of the action potential waveform. All data were performed with Neuromatic version 3.0 (http://www.neuromatic.thinkrandom.com) which runs within Igor pro 6.7.3.2 (WaveMetrics).
GST pulldown. GST-tagged NRG1-ICD (amino acid 279 to 644 in rat NRG1 I $\beta 1$ a proteins) were expressed in Escherichia coli BL21 cells and were purified using Glutathione Sepharose ${ }^{\mathrm{TM}} 4$ Fast Flow (GE Health) according to the manufacturer's instructions. His-tagged cytoplasmic loop (CL) 1 and 2 of SCN1A were purified using Ni-NTA agarose beads (QIAGEN) following the manufacturer's protocols. For binding assays, eluted His- $\mathrm{Na}_{\mathrm{v}} 1.1-\mathrm{CL}$ proteins were incubated with immobilized GST-NRG1-ICD or GST for $4 \mathrm{~h}$ at $4{ }^{\circ} \mathrm{C}$. The mixture was then washed, eluted, and subjected to western blot with anti-His and anti-GST antibodies (Abmart).

Stereotaxic injection of AAV. For virus injection, gtoNrg1 mice at age of $7-8$ weeks were anesthetized with $1 \%$ pentobarbital sodium $(100 \mathrm{mg} / \mathrm{kg}$, i.p.) and were placed in a stereotaxic apparatus (RWD Life Science). Viruses were injected bilaterally in the PFC (PrL and M2 cortex) with the coordinates: anteroposterior (AP) $2.34 \mathrm{~mm}$, mediolateral (ML) $\pm 0.75 \mathrm{~mm}$, dorsoventral (DV) $-2.00 \mathrm{~mm}$ relative to bregma. Each injection used $0.5 \mu \mathrm{lAV}$ and took $5 \mathrm{~min}$. After injection, the glass pipette was left in place for $5 \mathrm{~min}$ in order to facilitate diffusion of the virus. The injection sites were examined at the end of the experiments, and animals with incorrect injection sites were excluded from the data analysis. Three weeks after AAV injection, mice were subjected to experiments. All surgery was conducted with an aseptic technique. The AAV expressing TRE-hM3Dq-P2A-mCherry or TRE-EYFP were generated in OBiO Technology Corp., Ltd. The CNO is purchased from MCE company (HY-17366).

Statistical analysis. Two-way ANOVA was used in behavioral analysis including open field, PPI, and electrophysiological studies including I/O curve of AP, PPR, and $\mathrm{I} / \mathrm{V}$ curves of $\mathrm{Na}_{\mathrm{v}}$ channel activation. One-way ANOVA was used for the analysis of the data from three or more groups. Student's $t$ test was used to compare data from two groups. Data were expressed as mean \pm SEM unless otherwise indicated. The sample size justification was based on the previous studies ${ }^{21,26,40}$. According to the Wikipedia article on the normal distribution, about $95 \%$ of the values lie within two standard deviations. Our approach was to remove the data that were above (mean $+2 * \mathrm{SD})$ and below (mean $-2 * \mathrm{SD})$ before doing the statistical analysis. The example data shown were close to the overall mean. Statistically significant difference was indicated as follows: ${ }^{* * *} P<0.001,{ }^{* *} P<0.01$, and $* P<0.05$. The statistical analysis was performed with the software of GraphPad Prism 8.

Reporting summary. Further information on research design is available in the Nature Research Reporting Summary linked to this article.

\section{Data availability}

All data supporting the results presented herein are available from the article paper, Supplementary Information, and Source Data. The full-length images for all the gels or blots are provided in Supplementary Fig. 10. The web-links of databases GSE93577 and GSE93987 are as follows: https://www.ncbi.nlm.nih.gov/geo/query/acc.cgi? acc=GSE93577, https://www.ncbi.nlm.nih.gov/geo/query/acc.cgi?acc=GSE93987. All unique materials used are readily available from the corresponding author upon request. Source data are provided with this paper.

Received: 8 September 2020; Accepted: 8 December 2020; Published online: 12 January 2021

\section{References}

1. Marin, O. Interneuron dysfunction in psychiatric disorders. Nat. Rev. Neurosci. 13, 107-120 (2012).

2. Stefansson, H. et al. Neuregulin 1 and susceptibility to schizophrenia. Am. J. Hum. Genet 71, 877-892 (2002).

3. Stefansson, H. et al. Association of neuregulin 1 with schizophrenia confirmed in a Scottish population. Am. J. Hum. Genet. 72, 83-87 (2003).

4. Yang, J. Z. et al. Association study of neuregulin 1 gene with schizophrenia. Mol. Psychiatry 8, 706-709 (2003).

5. Mei, L. \& Nave, K. A. Neuregulin-ERBB signaling in the nervous system and neuropsychiatric diseases. Neuron 83, 27-49 (2014).

6. Mei, L. \& Xiong, W. C. Neuregulin 1 in neural development, synaptic plasticity and schizophrenia. Nat. Rev. Neurosci. 9, 437-452 (2008).

7. Wang, J. Y., Frenzel, K. E., Wen, D. \& Falls, D. L. Transmembrane neuregulins interact with LIM kinase 1, a cytoplasmic protein kinase implicated in development of visuospatial cognition. J. Biol. Chem. 273, 20525-20534 (1998)

8. Bertram, I. et al. Immunohistochemical evidence for impaired neuregulin-1 signaling in the prefrontal cortex in schizophrenia and in unipolar depression. Ann. N. Y Acad. Sci. 1096, 147-156 (2007). 
9. Parlapani, E. et al. Gene expression of neuregulin-1 isoforms in different brain regions of elderly schizophrenia patients. World J. Biol. Psychiatry 11, 243-250 (2010).

10. Hashimoto, R. et al. Expression analysis of neuregulin-1 in the dorsolateral prefrontal cortex in schizophrenia. Mol. Psychiatry 9, 299-307 (2004).

11. Law, A. J. et al. Neuregulin 1 transcripts are differentially expressed in schizophrenia and regulated by 5' SNPs associated with the disease. Proc. Natl Acad. Sci. USA 103, 6747-6752 (2006).

12. Hahn, C. G. et al. Altered neuregulin 1-erbB4 signaling contributes to NMDA receptor hypofunction in schizophrenia. Nat. Med. 12, 824-828 (2006).

13. Chong, V. Z. et al. Elevated neuregulin-1 and ErbB4 protein in the prefrontal cortex of schizophrenic patients. Schizophr. Res. 100, 270-280 (2008).

14. Weickert, C. S., Tiwari, Y., Schofield, P. R., Mowry, B. J. \& Fullerton, J. M. Schizophrenia-associated HapICE haplotype is associated with increased NRG1 type III expression and high nucleotide diversity. Transl. Psychiatry 2 , e104 (2012).

15. Liu, X. et al. Specific regulation of NRG1 isoform expression by neuronal activity. J. Neurosci. 31, 8491-8501 (2011).

16. Saunders, A. et al. Molecular diversity and specializations among the cells of the adult mouse brain. Cell 174, 1015-1030 e1016 (2018).

17. Zhong, S. et al. A single-cell RNA-seq survey of the developmental landscape of the human prefrontal cortex. Nature 555, 524-528 (2018).

18. Lewis, D. A. \& Sweet, R. A. Schizophrenia from a neural circuitry perspective: advancing toward rational pharmacological therapies. J. Clin. Investig. 119, 706-716 (2009).

19. Birnbaum, R. \& Weinberger, D. R. Genetic insights into the neurodevelopmental origins of schizophrenia. Nat. Rev. Neurosci. 18, 727-740 (2017).

20. Mirnics, K. \& Pevsner, J. Progress in the use of microarray technology to study the neurobiology of disease. Nat. Neurosci. 7, 434-439 (2004).

21. Enwright Iii, J. F. et al. Transcriptome alterations of prefrontal cortical parvalbumin neurons in schizophrenia. Mol. Psychiatry 23, 1606-1613 (2018).

22. Arion, D. et al. Distinctive transcriptome alterations of prefrontal pyramidal neurons in schizophrenia and schizoaffective disorder. Mol. Psychiatry 20, 1397-1405 (2015).

23. Benzel, I. et al. Interactions among genes in the ErbB-Neuregulin signalling network are associated with increased susceptibility to schizophrenia. Behav. Brain Funct. 3, 31 (2007).

24. Wang, Y. C. et al. Neuregulin 3 genetic variations and susceptibility to schizophrenia in a Chinese population. Biol. Psychiatry 64, 1093-1096 (2008).

25. Chen, P. L. et al. Fine mapping on chromosome 10q22-q23 implicates Neuregulin 3 in schizophrenia. Am. J. Hum. Genet. 84, 21-34 (2009).

26. Yin, D. M. et al. Reversal of behavioral deficits and synaptic dysfunction in mice overexpressing neuregulin 1. Neuron 78, 644-657 (2013).

27. Tanaka, K. F. et al. Expanding the repertoire of optogenetically targeted cells with an enhanced gene expression system. Cell Rep. 2, 397-406 (2012).

28. Geyer, M. A. \& Braff, D. L. Startle habituation and sensorimotor gating in schizophrenia and related animal models. Schizophr. Bull. 13, 643-668 (1987).

29. Corcoran, R., Mercer, G. \& Frith, C. D. Schizophrenia, symptomatology and social inference: investigating "theory of mind" in people with schizophrenia. Schizophr. Res. 17, 5-13 (1995).

30. Hoftman, G. D., Datta, D. \& Lewis, D. A. Layer 3 excitatory and inhibitory circuitry in the prefrontal cortex: developmental trajectories and alterations in schizophrenia. Biol. Psychiatry 81, 862-873 (2017).

31. Tumbar, T. et al. Defining the epithelial stem cell niche in skin. Science 303, 359-363 (2004).

32. Whittington, M. A., Faulkner, H. J., Doheny, H. C. \& Traub, R. D. Neuronal fast oscillations as a target site for psychoactive drugs. Pharm. Ther. 86, 171-190 (2000).

33. Yu, F. H. \& Catterall, W. A. Overview of the voltage-gated sodium channel family. Genome Biol. 4, 207 (2003).

34. Cheah, C. S. et al. Specific deletion of NaV1.1 sodium channels in inhibitory interneurons causes seizures and premature death in a mouse model of Dravet syndrome. Proc. Natl Acad. Sci. USA 109, 14646-14651 (2012).

35. Fazzari, P. et al. Control of cortical GABA circuitry development by $\mathrm{Nrg} 1$ and ErbB4 signalling. Nature 464, 1376-1380 (2010).

36. Vullhorst, D. et al. Selective expression of ErbB4 in interneurons, but not pyramidal cells, of the rodent hippocampus. J. Neurosci. 29, 12255-12264 (2009).

37. Yau, H. J., Wang, H. F., Lai, C. \& Liu, F. C. Neural development of the neuregulin receptor ErbB4 in the cerebral cortex and the hippocampus: preferential expression by interneurons tangentially migrating from the ganglionic eminences. Cereb. Cortex 13, 252-264 (2003).

38. Woo, R. S. et al. Neuregulin-1 enhances depolarization-induced GABA release. Neuron 54, 599-610 (2007).

39. Wen, L. et al. Neuregulin 1 regulates pyramidal neuron activity via ErbB4 in parvalbumin-positive interneurons. Proc. Natl Acad. Sci. USA 107, 1211-1216 (2010).
40. Chen, Y. J. et al. ErbB4 in parvalbumin-positive interneurons is critical for neuregulin 1 regulation of long-term potentiation. Proc. Natl Acad. Sci. USA 107, 21818-21823 (2010)

41. Catterall, W. A. From ionic currents to molecular mechanisms: the structure and function of voltage-gated sodium channels. Neuron 26, 13-25 (2000).

42. Roth, B. L. DREADDs for Neuroscientists. Neuron 89, 683-694 (2016).

43. Marissal, T. et al. Restoring wild-type-like CA1 network dynamics and behavior during adulthood in a mouse model of schizophrenia. Nat. Neurosci. 21, 1412-1420 (2018).

44. Mukherjee, A., Carvalho, F., Eliez, S. \& Caroni, P. Long-lasting rescue of network and cognitive dysfunction in a genetic schizophrenia model. Cell 178, 1387-1402 e1314 (2019).

45. Bast, T., Zhang, W. N. \& Feldon, J. Hyperactivity, decreased startle reactivity, and disrupted prepulse inhibition following disinhibition of the rat ventral hippocampus by the GABA(A) receptor antagonist picrotoxin. Psychopharmacology 156, 225-233 (2001).

46. Enomoto, T., Tse, M. T. \& Floresco, S. B. Reducing prefrontal gammaaminobutyric acid activity induces cognitive, behavioral, and dopaminergic abnormalities that resemble schizophrenia. Biol. Psychiatry 69, 432-441 (2011).

47. Israelashvili, M., Yael, D., Vinner, E., Belelovsky, K. \& Bar-Gad, I. Common neuronal mechanisms underlying tics and hyperactivity. Cortex 127, 231-247 (2020).

48. Pezze, M., McGarrity, S., Mason, R., Fone, K. C. \& Bast, T. Too little and too much: hypoactivation and disinhibition of medial prefrontal cortex cause attentional deficits. J. Neurosci. 34, 7931-7946 (2014).

49. Agarwal, A. et al. Dysregulated expression of neuregulin-1 by cortical pyramidal neurons disrupts synaptic plasticity. Cell Rep. 8, 1130-1145 (2014).

50. Deakin, I. H. et al. Transgenic overexpression of the type I isoform of neuregulin 1 affects working memory and hippocampal oscillations but not long-term potentiation. Cereb. Cortex 22, 1520-1529 (2012).

51. Olaya, J. C. et al. Overexpression of neuregulin 1 type III confers hippocampal mRNA alterations and schizophrenia-like behaviors in mice. Schizophr. Bull. 44, 865-875 (2018).

52. Deakin, I. H. et al. Altered hippocampal gene expression and structure in transgenic mice overexpressing neuregulin 1 (Nrg1) type I. Transl. Psychiatry 8, 229 (2018).

53. Luo, X., He, W., Hu, X. \& Yan, R. Reversible overexpression of bace1-cleaved neuregulin-1 N-terminal fragment induces schizophrenia-like phenotypes in mice. Biol. Psychiatry 76, 120-127 (2014).

54. Papaleo, F. et al. Behavioral, neurophysiological, and synaptic impairment in a transgenic neuregulin1 (NRG1-IV) murine schizophrenia model. J. Neurosci. 36, 4859-4875 (2016)

55. Kotzadimitriou, D. et al. Neuregulin 1 type I overexpression is associated with reduced NMDA receptor-mediated synaptic signaling in hippocampal interneurons expressing PV or CCK. eNeuro 5, e0418-17.2018 1-18 (2018).

56. Sun, Y. et al. Neuregulin-1/ErbB4 signaling regulates visual cortical plasticity. Neuron 92, 160-173 (2016).

57. Grieco, S. F. et al. Subanesthetic ketamine reactivates adult cortical plasticity to restore vision from amblyopia. Curr. Biol. 30, 3591-3603 (2020).

58. Sponheim, S. R., Clementz, B. A., Iacono, W. G. \& Beiser, M. Resting EEG in first-episode and chronic schizophrenia. Psychophysiology 31, 37-43 (1994).

59. Lee, K. H., Williams, L. M., Breakspear, M. \& Gordon, E. Synchronous gamma activity: a review and contribution to an integrative neuroscience model of schizophrenia. Brain Res. Brain Res. Rev. 41, 57-78 (2003).

60. Flames, N. et al. Short- and long-range attraction of cortical GABAergic interneurons by neuregulin-1. Neuron 44, 251-261 (2004)

61. Ting, A. K. et al. Neuregulin 1 promotes excitatory synapse development and function in GABAergic interneurons. J. Neurosci. 31, 15-25 (2011).

62. Cahill, M. E. et al. Control of interneuron dendritic growth through NRG1/ erbB4-mediated kalirin-7 disinhibition. Mol. Psychiatry 17(1), 99-107 (2012).

63. Del Pino, I. et al. Erbb4 deletion from fast-spiking interneurons causes schizophrenia-like phenotypes. Neuron 79, 1152-1168 (2013).

64. Tan, G. H. et al. Neuregulin 1 represses limbic epileptogenesis through ErbB4 in parvalbumin-expressing interneurons. Nat. Neurosci. 15, 258-266 (2011).

65. Yin, D. M. et al. Calcyon stimulates neuregulin 1 maturation and signaling. Mol. Psychiatry 20, 1251-1260 (2015).

66. Li, K. X. et al. Neuregulin 1 regulates excitability of fast-spiking neurons through Kv1.1 and acts in epilepsy. Nat. Neurosci. 15, 267-273 (2011).

67. Janssen, M. J., Leiva-Salcedo, E. \& Buonanno, A. Neuregulin directly decreases voltage-gated sodium current in hippocampal ErbB4-expressing interneurons. J. Neurosci. 32, 13889-13895 (2012).

68. Bao, J., Wolpowitz, D., Role, L. W. \& Talmage, D. A. Back signaling by the Nrg-1 intracellular domain. J. Cell Biol. 161, 1133-1141 (2003). 
69. Chen, Y., Hancock, M. L., Role, L. W. \& Talmage, D. A. Intramembranous valine linked to schizophrenia is required for neuregulin 1 regulation of the morphological development of cortical neurons. J. Neurosci. 30, 9199-9208 (2010).

70. Fazzari, P. et al. Cell autonomous regulation of hippocampal circuitry via Aphlb-gamma-secretase/neuregulin 1 signalling. eLife 3, e02196 (2014).

71. Smith, R. D. \& Goldin, A. L. Phosphorylation of brain sodium channels in the I-II linker modulates channel function in Xenopus oocytes. J. Neurosci. 16, 1965-1974 (1996).

72. Citri, A., Pang, Z. P., Sudhof, T. C., Wernig, M. \& Malenka, R. C. Comprehensive qPCR profiling of gene expression in single neuronal cells. Nat. Protoc. 7, 118-127 (2011).

73. $\mathrm{Yu}, \mathrm{Q}$. et al. Genetic labeling reveals temporal and spatial expression pattern of D2 dopamine receptor in rat forebrain. Brain Struct. Funct. 224, 1035-1049 (2019).

74. Deacon, R. M. Assessing nest building in mice. Nat. Protoc. 1, 1117-1119 (2006).

75. Lin, L. et al. Large-scale neural ensemble recording in the brains of freely behaving mice. J. Neurosci. Methods 155, 28-38 (2006).

\section{Acknowledgements}

This work was supported by grants from National Natural Science Foundation of China (No. 81471118 and 31861143033); grants from Shanghai Key Laboratory of Psychotic Disorders (No. 13dz2260500); grants from State Key Laboratory of Neuroscience. Dr. Dong-Min Yin is a NARSAD Young Investigator. We thank Dr. Yuchio Yanagawa for proving Gad67-tTA mice.

\section{Author contributions}

Y.-Y.W. performed biochemical and behavioral experiments. B.Z. performed the electrophysiology recordings in brain slices. M.-M.W. and L.L. performed in vivo recordings. X.-L.Z. assisted the biochemical experiments. Y.-Y.W., B.Z., L.L., and D.-M.Y. analyzed the data. D.-M.Y. designed the experiments, supervised the work, and wrote the paper.

\section{Competing interests}

The authors declare no competing interests.

\section{Additional information}

Supplementary information is available for this paper at https://doi.org/10.1038/s41467020-20552-y.

Correspondence and requests for materials should be addressed to D.-M.Y.

Peer review information Nature Communications thanks Tim Karl and other, anonymous, reviewers for their contributions to the peer review of this work. Peer review reports are available.

Reprints and permission information is available at http://www.nature.com/reprints

Publisher's note Springer Nature remains neutral with regard to jurisdictional claims in published maps and institutional affiliations.

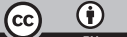

Open Access This article is licensed under a Creative Commons Attribution 4.0 International License, which permits use, sharing, adaptation, distribution and reproduction in any medium or format, as long as you give appropriate credit to the original author(s) and the source, provide a link to the Creative Commons license, and indicate if changes were made. The images or other third party material in this article are included in the article's Creative Commons license, unless indicated otherwise in a credit line to the material. If material is not included in the article's Creative Commons license and your intended use is not permitted by statutory regulation or exceeds the permitted use, you will need to obtain permission directly from the copyright holder. To view a copy of this license, visit http://creativecommons.org/ licenses/by/4.0/.

(C) The Author(s) 2021 